\title{
Heavy resonances at energy-frontier hadron colliders
}

\author{
Clement Helsens $^{1, \mathrm{a}}{ }_{\mathbb{O}}$, David Jamin ${ }^{1,3, \mathrm{~b}}$, Michelangelo L. Mangano ${ }^{1, \mathrm{c}}$, Thomas G. Rizzo ${ }^{2, \mathrm{~d}}$, Michele Selvaggi ${ }^{1, \mathrm{e}}$ \\ ${ }^{1}$ CERN, 1211 Geneva 23, Switzerland \\ 2 SLAC National Accelerator Laboratory, 2575 Sand Hill Rd., Menlo Park, CA 94025, USA \\ ${ }^{3}$ Academia Sinica, Institute of Physics, Taipei, Taiwan
}

Received: 11 March 2019 / Accepted: 20 June 2019 / Published online: 8 July 2019

(C) The Author(s) 2019

\begin{abstract}
This paper explores the physics reach of the proton-proton future circular collider (FCC-hh) and of the High-Energy LHC (HE-LHC) for searches of new particles produced in the $s$-channel and decaying to two high-energy leptons, jets (non-tops), tops or W/Z bosons. We discuss the expected discovery potential and exclusion limits for benchmark models predicting new massive particles that result in resonant structures in the invariant mass spectrum. We also present a detailed study of the HE-LHC potential to discriminate among different models, for a $Z^{\prime}$ that could be discovered by the end of high-luminosity LHC (HL-LHC).
\end{abstract}

\section{Contents}

1 Introduction . . . . . . . . . . . . 1

2 BSM $Z^{\prime}$ models and their possible probes . . . . . . 2

3 Simulation setup ............... . . . 4

3.1 Monte Carlo production . . . . . . . . . . . 4

3.2 Simulation of the detector response . . . . . . . 4

3.2 .1 Tracking ............ 4

3.2.2 Calorimetry and particle-flow . . . . 5 5

3.2.3 Object identification efficiencies . . . . . 6

3.3 Treatment of the Monte Carlo samples . . . . . 6

3.4 Statistical analysis . . . . . . . . . . . . . . 6

4 Studies at $100 \mathrm{TeV} \ldots \ldots \ldots \ldots$

4.1 Leptonic final states . . . . . . . . . . . . 7

4.1.1 The $e^{+} e^{-}$and $\mu^{+} \mu^{-}$final states . . . . 7

4.1.2 The $\tau^{+} \tau^{-}$final state . . . . . . . . . 7

4.1.3 Sensitivity to flavour-anomaly inspired $Z^{\prime}$ models ............ 8

\footnotetext{
a e-mail: clement.helsens@cern.ch

b e-mail: david.jamin @ cern.ch

c e-mail: michelangelo.mangano@cern.ch

de-mail: rizzo@slac.stanford.edu

ee-mail: michele.selvaggi@cern.ch
}

4.2 Hadronic final states . . . . . . . . . . . . . 9

4.2.1 Multi-variate object tagging . . . . . . 9 9

4.2.2 The $j j$ final state . . . . . . . . . 11

4.2.3 The $t \bar{t}$ final state . . . . . . . . . . . 11

4.2.4 The $W^{+} W^{-}$final state . . . . . . . . 11

5 Comparison with the $27 \mathrm{TeV}$ HE-LHC . . . . . 12

6 Characterisation of a $Z^{\prime}$ discovery . . . . . . . . . 14

6.1 Context of the study . . . . . . . . . . . . . 14

6.2 Bounds from HL-LHC . . . . . . . . . . . 14

6.3 Definition of the discriminating variables . . . 14

6.3.1 Leptonic final states . . . . . . . . 15

6.3.2 Hadronic final states . . . . . . . . . 15

7 Conclusions ................ . . 16

Appendix A: Discussion of the detector performance . 17

Appendix B: Tagging rate function . . . . . . . . . 18

Appendix C: Background fit . . . . . . . . . . . . . . 19

Appendix D: Multivariate object tagger . . . . . . . . 19

Appendix E: Summary plots . . . . . . . . . . . . . 19

References . . . . . . . . . . . . . . . 22

\section{Introduction}

Extensive searches at the LHC, addressing a broad range of final states, have set stringent limits on the existence of new physics beyond the Standard Model (SM). While the LHC has still a long way to go in exposing new phenomena [1], several projects are being proposed for future colliders, whose principal goal is to push even further the sensitivity to the theoretical scenarios beyond the SM (BSM) that have been proposed to address the shortcomings of the SM. These range from the theoretical puzzle of hierarchy problem, to the limitations of the SM to account for observed phenomena such as dark matter, neutrino masses or the matter-antimatter asymmetry of the universe. These future projects are designed to address the possible reasons for the current lack of BSM evidence: either the BSM phenomena appear at energy scales 
consistent with the reach of the LHC, but elude the LHC searches due to the stealthy nature of their final states or to very weak couplings leading to very low rates, or the BSM phenomena are tied to physics living at mass scales beyond the LHC reach. Future $e^{+} e^{-}$colliders have a limited energy reach for direct observation, but their clean experimental environment and high precision have the potential to expose the most elusive manifestations of TeV-scale BSM models. Future pp colliders, on the other hand, allow us to extend the direct discovery reach to masses well beyond those probed by the LHC, and their high luminosity may give visibility to the rarest of the processes. The HE-LHC [2] is designed to increase the LHC energy to $27 \mathrm{TeV}$, thus almost doubling the LHC mass reach. The FCC-hh $[3,4]$ and the SPPC [5,6] are designed to reach a pp centre of mass energy of $100 \mathrm{TeV}$, relying on new facilities built around a $100 \mathrm{~km}$ accelerator ring, with a potential increase in mass reach of a factor of 5-7, depending on their integrated luminosity and on the production channel. In addition to greatly extending the discovery reach, future $\mathrm{pp}$ colliders would enhance the production rate of new particles that the LHC could still discover during its forthcoming operations. This would enable a much more precise study of the properties of these new particles, and help to pin down the underlying physics models that will extend the SM.

General overviews of the BSM discovery potential of HELHC and FCC-hh, spanning across a broad range of models, can be found in Refs. [1,7]. This paper focuses on a quantitative study of the discovery potential at the highest masses, using as benchmarks $s$-channel resonances. Section 2 presents the expectations of some relevant BSM scenarios, and defines the models that we shall discuss. Details on the generators, detector performance, statistical method and other analysis techniques are presented in Sect. 3. The leptonic (ee, $\mu \mu, \tau \tau)$ and the hadronic ( WW, $t \bar{t}$ and jj) analyses are detailed in Sects. 4.1 and 4.2 respectively. The results of the analyses are discussed in the context of the anticipated FCC-hh accelerator and detector performance; the sensitivity of the HE-LHC to the same benchmark models is summarised in Sect. 5. Finally, a study of $Z^{\prime}$ models' discrimination at the HE-LHC, in case of a discovery at the HL-LHC, is presented in Sect. 6. The work in this paper has been conducted during the preparation of the FCC conceptual design reports Volume 1 and $4[3,4]$ and in the report from working group 3 on the Physics of the HL-LHC, and Perspectives at the HE-LHC [1]. This article provides a detailed description of the key results summarized in those documents.

\section{BSM $Z^{\prime}$ models and their possible probes}

In order to explore and contrast the capabilities of future colliders to discover and examine the properties of possi- ble new physics, a broad set of benchmark models needs to be studied. In the case of new heavy resonances, this benchmark set should be sufficiently complete that all of the major discovery channels of relevance are represented. Here we are particularly interested in the 2-body final states of these resonances, consisting of opposite sign dilepton pairs $\left(e^{+} e^{-}, \mu^{+} \mu^{-}\right.$and $\left.\tau^{+} \tau^{-}\right)$, dijets, $t \bar{t}$ and $W^{+} W^{-}$. We note that typically at least one or possibly more of these 2-body channels will possess a significant branching fraction, particularly into jets, given the partonic production mechanism. Note that decays into pairs of secondary objects that then themselves decay hadronically can often populate the dijet channel if the final state jets are sufficiently boosted. The dijet channel can thus represent many different final states, unless substructure studies are performed. When there are 2 or more of these channels available for simultaneous study we have an increased chance to learn more about the underlying physics model. The most important properties of a newly discovered resonance that need to be determined (other than the mass) are its production cross section, which, especially for a broad resonance, will sometimes require a good understanding of the underlying background shape, and its spin (as was the case of the Higgs boson). These properties alone can provide important information about the BSM model from which the signal originated. The spin measurement usually requires the reconstruction of the angular distribution of the resonance decay products and, hence, a respectable amount of statistics, although the observation of certain final states can immediately exclude some spin possibilities as was the case with the observation of $H \rightarrow \gamma \gamma$.

A new, neutral, spin-1 gauge boson, $Z^{\prime}$, which is usually a color-singlet object produced in the $q \bar{q}$ channel, is a ubiquitous feature of many BSM models [8-11]. While falling into several distinct classes, $Z^{\prime}$ are most commonly associated with the extension of the SM EW gauge group by an additional $\mathrm{U}(1)$ or $\mathrm{SU}(2)$ factor, although more significant additions are possible. When the additional factor is nonabelian, as in the case of $\mathrm{SU}(2)$, a new $W^{ \pm}$, gauge boson also appears in the spectrum together with the $Z^{\prime}$, and with a comparable mass. Of this subset of models, those that arise from Grand Unified Theory frameworks are the ones most commonly encountered in the literature, and include familiar examples such as the left-right symmetric model (LRM) $[12,13]$ which results from $\mathrm{SO}(10)$ (or larger GUT groups) and where the $\mathrm{SM}$ is augmented by an $\mathrm{SU}(2)_{R}$ factor. For example, the LRM can arise from $\mathrm{SO}(10)$ breaking at the GUT scale directly to $\mathrm{SU}(2)_{L} \times \mathrm{SU}(2)_{R} \times \mathrm{U}(1)_{B}-L$, which then breaks to the SM at a few to multi-TeV scale. A second set of GUT-based $Z^{\prime}$ models arise from $E_{6}$ [14-17], where, e.g., $E_{6} \rightarrow S M \times U(1)_{\psi} \times \mathrm{U}(1)_{\chi} \rightarrow \mathrm{SM} \times \mathrm{U}(1)_{\theta}$, giving rise to an additional $\mathrm{U}(1)_{\theta}$ gauge group. Note that here $\theta$ labels the linear combination of $\mathrm{U}(1)_{\psi}-\mathrm{U}(1)_{\chi}$ that remains unbroken to energies below the GUT scale. A common set 
of features of this GUT-based class of models include their generation-universal couplings of the $Z^{\prime}$ to the SM fermions, their charges commuting with those of the SM, so that, e.g., $u_{L}$ and $d_{L}$ have the same $Z^{\prime}$ coupling and the resonances themselves are usually narrow, reflecting EW strength or weaker couplings with width to mass ratios $\Gamma / M<0.01-$ 0.03. In particular, the GUT origin of these models implies that this class of $Z^{\prime}$ can be used to simultaneously study all of the dileptonic channels: $e^{+} e^{-}, \mu^{+} \mu^{+}$as well as $\tau^{+} \tau^{-}$ together with the dijets, $W^{+} W^{-}$and $t \bar{t}$ channels as well.

With this much information potentially available from the observation of a given $Z^{\prime}$ in multiple channels, one may try to distinguish it from another $Z^{\prime}$ of similar type, given sufficient statistics and well-controlled systematics. In addition to relative cross section measurements, e.g., that of dijets and/or $t \bar{t}$ compared to dileptons, the cleanliness of the dilepton channel itself provides additional information. Since the lepton charges can be determined, their angular distribution allows us to probe the forward-backward asymmetry, $A_{F B}$, whose sensitivity to the quark and lepton couplings is complementary to that of the dilepton production cross section. ${ }^{1}$ A second handle [18] to probe the couplings to the various quark flavours is the different rapidity distributions of the $u \bar{u}$ and $d \bar{d}$ initial states. Since the various $Z^{\prime}$ will generally couple differently to the $u$ and $d$ quarks, the rapidity distributions of the dilepton final state will probe these coupling variations. The relevant observable in this case is the rapidity ratio, $r_{y}$, defined by the ratio of the number of dilepton pairs produced at central versus forward rapidities (see below for details).

Returning to our discussion of these specific GUT-inspired models, we note that in the LRM with the assumption of leftand right-handed gauge couplings, i.e., $\kappa=g_{R} / g_{L}=1$, all of the various interactions of the $Z^{\prime}$ with the SM fields are completely fixed. However, in the $E_{6}$ model case, the single new mixing parameter, $\theta$, controls the couplings of the $Z^{\prime}$ to the various SM particles; four particular choices for the value of this parameter correspond to the more specific model cases discussed here and are denoted as $\psi, \chi, \eta$ and I. As in the SM, the $Z^{\prime}$ in GUT models generally couple to all the familiar quarks and leptons and thus can easily populate simultaneously the various fermionic 2-body final states listed above at various predictable rates. The measurement of these rates (as well as other associated observables) can be then used to discriminate among the different $Z^{\prime}$ possibilities after discovery, as will be discussed further below.

\footnotetext{
1 The scattering angle is defined by the direction of the outgoing charged lepton w.r.t. the incoming quark direction. For the heaviest resonances, where the quark is dominantly a valence parton inside the proton, the latter is usually also the direction of the boost of the $Z^{\prime}$ in the lab frame. We adopt this convention in our analysis, using the Monte Carlo to correct on average for the dilution of $A_{F B}$ induced by antiquark contributions.
}

Note that the decay rate for $Z^{\prime}$ into the $W^{+} W^{-}$final state in GUT frameworks is highly dependent on the details of the model building assumptions within a specific scenario and especially upon the detailed nature of spontaneous symmetry breaking as manifested by the amount of mixing (if it occurs at all) between the $Z^{\prime}$ and SM $Z$; the $Z^{\prime}$ coupling to $W^{+} W^{-}$in $\mathrm{U}(1)$ extensions is always controlled solely by the amount of this gauge boson mixing, i.e., this coupling in such extensions in the absence of mixing is zero.

The $Z^{\prime}$ of the Sequential Standard Model [19] (SSM) has been used very frequently for many years as a standard candle by experimenters, since it conveniently posits the existence of heavier copies of the SM gauge bosons, with heavier masses but identical couplings; this provides a useful yardstick for easier performance comparisons.

Alternative models of EW symmetry breaking, including the topcolor assisted technicolor scenarios [20], also lead frequently to $Z^{\prime}$-like states with resonance signatures. The greatest difference of such theories from the GUT-type $Z^{\prime}$ models lies in their generation-dependent couplings, potentially of QCD strength. (The color-octet versions of such states in this model class are called colorons.) This implies that the corresponding resonances will likely not be narrow and will preferentially couple, by construction, to the third generation, leading to highly boosted $t \bar{t}$ final states, and proving a useful benchmark model for this channel. Similar new $Z^{\prime}$ states can also arise in Little Higgs models [21], which also have preferential decays to third generation states.

Occasionally, the expected properties of a new $Z^{\prime}$ models are suggested by the attempt to interpret and model anomalies observed in the data. For example, a $Z^{\prime}$ with an unusual flavor-dependent coupling structure has recently been suggested as a (partially complete) UV model to explain the apparent anomaly seen in semileptonic $b \rightarrow s l^{+} l^{-}$decays $[22,23]$. In effective field theory language, a new interaction of the form $\sim \bar{b} \gamma_{\mu} P_{L} s \bar{\mu} \gamma^{\mu} P_{L} \mu$ of proper strength can provide a reasonable fit to these experimental observations [24]. This operator can be induced by the exchange of a heavy $Z^{\prime}$ potentially accessible to high energy colliders [25,26]. This $Z^{\prime}$, in the weak basis, couples only to the third generation quark doublet and to the muon lepton doublet, so that it will have a suppressed production cross section at hadron colliders. Such a $Z^{\prime}$ could be observed in both the dimuon and ditop channels.

Models of composite quarks and leptons offer another path wherein new resonances are predicted. Excited quarks $[27,28], Q^{*}$, are spin-1/2, color triplet states with the same SM quantum numbers of quarks. There is, as of yet, no fundamental, UV-complete model encompassing this idea so that this framework is purely phenomenological. The SM quarks couple to these excited states via a magnetic-dipole-like interaction together with an associated SM gauge boson $(g, \gamma$ or $W / Z$ ). This dimension-5 interaction is suppressed by a large 
'compositeness scale', $\Lambda$, and the relative coupling strengths to the different gauge bosons are partially controlled by a set of essentially free parameters, $f_{i}$. Excited quarks can be singly produced in the $g q$ channel, to which they will also dominantly decay due to the presence of the strong coupling constant, yielding the dijet signature of interest to us here (the $q \gamma$ channel is also possible, but will not be considered here). It is useful to have a benchmark model with dijet decays which take place in the $g q$ channel (as opposed to a $Z^{\prime}$ that can only populate the $q \bar{q}$ dijet channel) with which to compare and contrast. The angular distributions of the 2 jets in the dijet decay, which will require significant statistics to determine, can provide us information about the spin of the original resonance and the nature of its couplings to the decay products [29-32].

Spin-2 graviton resonances occur in extra-dimensional scenarios that attempt to address the hierarchy problem, as in the case of the warped extra dimensional model of Randall and Sundrum (RS) [33]. In such setups, the SM gauge fields and fermions are generally allowed to propagate in the 5-dimensional bulk [34-38] whereas EW symmetry breaking occurs at or near the TeV/SM brane via the usual Higgs mechanism. Due to the shape of their 5-D wavefunctions, the Kaluza-Klein excitations of the familiar graviton, $G_{R S}$ [39] will dominantly decay into objects localized near to where SM symmetry breaking occurs, i.e., Higgs boson pairs and $t \bar{t}$, as well as to the longitudinal components of the massive SM gauge bosons, e.g., $W_{L}^{+} W_{L}^{-}$, all with relatively fixed branching factions with only some small allowed variations. Thus $G_{R S} \rightarrow W^{+} W^{-}$in the RS framework provides an excellent benchmark model for the study of resonant and highly boosted $W$-pairs. For hadronic $W$ decays, given the high boost, this final state may also (appear to) populate the resonant dijet channel. One notes that apart from the $G_{R S}$ mass scale itself, essentially the only other free parameter in this RS model setup (wherein the lighter fermions are essentially decoupled from the graviton resonances), is frequently denoted by $c=k / \bar{M}_{P l}$, which simply controls the overall coupling strength to all of the various SM particles.

\section{Simulation setup}

\subsection{Monte Carlo production}

Monte Carlo (MC) event samples are used to simulate the response of the detector to signals and backgrounds processes. Signal events are generated with PYTHIA8 [40] version 8.201 with the NNPDF2.3NNLO PDF set [41] using the leading order cross-section from the generator with no $\mathrm{K}$ factor. The SM backgrounds considered are Drell-Yan, dijet (QCD), top pairs $(t \bar{t}), V V$ and $\mathrm{V}+$ jets where $V=W / Z$ and were generated using MG5_aMC [42] version 2.5.5 at leading order only with the NNPDF3.0NLO [41] PDF set in bins of $H_{T}$. A K-factor of 2 is applied to all the background processes to account for higher order corrections and is considered to be very conservative.

\subsection{Simulation of the detector response}

This study discusses the discovery potential of heavy resonances decaying to multi-TeV final states. The ability to accurately reconstruct highly boosted final states is largely dependent on the nature of the object and on the detector assumptions. Generally speaking, the energy-momentum resolution of calorimetric objects such as electrons, photons and jets improves as a function of the energy. Conversely, the momentum resolution of charged particles reconstructed as tracks decreases with the momentum as the curvature of the trajectory vanishes. In addition, at high energies, composite objects such as jets, or hadronically decaying $\tau$ 's and heavy bosons are highly collimated. This results in an effectively coarser granularity of the detector, which can potentially limit the ability to resolve and identify the decay products inside the jets, thereby limiting the identification and QCD background rejection capabilities.

The detector response has been simulated via the DELPHES software package [43]. For the $\sqrt{s}=100 \mathrm{TeV}$ collider, the reference FCC-hh detector configuration has been used as a baseline $[3,44]$. For the HE-LHC study with $\sqrt{s}=27 \mathrm{TeV}$ a generic detector configuration [45] has been designed to reproduce an average response of the HL-LHC general purpose ATLAS [46,47] and CMS [48] detectors. Hereafter, we will simply refer to the HE-LHC and the FCChh detectors and will only discuss detector specifications that are relevant for high $p_{\mathrm{T}}$ objects. The overall contribution of pile-up is neglected altogether, as it is expected to have a negligible impact of multi-TeV objects. Even though only one specific configuration of the detector response has been studied in details for FCC-hh, the effect of the degradation of some key parameters have been examined and is documented in Appendix A.

\subsubsection{Tracking}

After collision, parton showering, hadronisation, and decays, the first step of DELPHES is the propagation of long-lived particles inside the tracking volume within a uniform axial magnetic field parallel to the beam direction. The magnetic field strength $B$, the size of the tracking radius, $L$, and the single hit spatial resolution, $\sigma_{r \phi}$, are the main parameters that determine the resolution on the track transverse momentum:

$$
\frac{\sigma\left(p_{\mathrm{T}}\right)}{p_{\mathrm{T}}} \approx \frac{\sigma_{r \phi} p_{\mathrm{T}}}{B \cdot L^{2}} .
$$


Table 1 Tracking-related parameters for the FCC-hh and HE-LHC detectors in Delphes

\begin{tabular}{lll}
\hline & FCC-hh & HE-LHC \\
\hline$B_{z}(T)$ & 4 & 4 \\
Length $(m)$ & 10 & 6 \\
Radius $(m)$ & 1.5 & 1.1 \\
$\epsilon$ & 0.95 & 0.95 \\
$\sigma(\eta, \phi)(m r a d)$ & 1 & 3 \\
$\sigma\left(p_{\mathrm{T}}\right) / p_{\mathrm{T}}$ (tracks) & $0.02 \cdot p_{\mathrm{T}}(\mathrm{TeV} / \mathrm{c})$ & $0.1 \cdot p_{\mathrm{T}}(\mathrm{TeV} / \mathrm{c})$ \\
$\sigma\left(p_{\mathrm{T}}\right) / p_{\mathrm{T}}=5 \%$ (muons) & $p_{\mathrm{T}}=15 \mathrm{TeV}$ & $p_{\mathrm{T}}=2 \mathrm{TeV}$ \\
\hline
\end{tabular}

The radius of the FCC-hh inner tracking detector is $3 / 2$ that of the HE-LHC detector with a similar magnetic field of $4 \mathrm{~T}$. The spatial resolution $\sigma_{r \phi}$ is 3 times smaller than at HE-LHC, which is possible thanks to a more granular pixel detector [49]. These specifications of the FCC-hh detector would allow measurements of $p_{\mathrm{T}}=1 \mathrm{TeV}$ charged hadrons with a precision of $\sigma\left(p_{\mathrm{T}}\right) / p_{\mathrm{T}} \simeq 2 \%$, compared to $\sigma\left(p_{\mathrm{T}}\right) / p_{\mathrm{T}} \simeq 10 \%$ for the HE-LHC detector.

Central and isolated high momentum charged hadron tracks are assumed to be reconstructed with an efficiency $\epsilon=95 \%$. However, charged particles confined inside a highly boosted jet can be extremely collimated, resulting in unresolvable tracker hits, especially in the innermost tracking layers. Although an accurate description of this feature would require a full event reconstruction by means of a GEANT4based simulation [50-52], a specific DELPHES module aiming at reproducing this effect has been designed. Whenever two or more tracks fall within an angular separation $\sigma(\eta, \phi)$, only the highest momentum track is reconstructed. This effect can result in an additional inefficiency to that shown in Table 1, and can affect the ability to reconstruct tracks in the core of highly boosted jets, as shown in Fig. 1 (left).

Muons are also reconstructed using tracking. However, an additional stand-alone muon measurement is provided by the angular difference between track angle in the muon system and the radial line connection to the beam axis, giving a large improvement on the resolution at high $p_{\mathrm{T}}$ [3]. Assuming a 2 times better position resolution of the muon system for the FCC-hh detector, a combined muon momentum resolution of $\sigma\left(p_{\mathrm{T}}\right) / p_{\mathrm{T}} \simeq 5 \%$ can be achieved for momenta as high as $p_{\mathrm{T}}=15 \mathrm{TeV}$, as opposed to $p_{\mathrm{T}}=2 \mathrm{TeV}$ for the HE-LHC detector.

\subsubsection{Calorimetry and particle-flow}

After propagating within the magnetic field, long-lived particles reach the electromagnetic (ECAL) and hadronic (HCAL) calorimeters. Since these are modeled in DELPHES by two-dimensional grids of variable spacing, the calorimeter deposits natively include finite angular resolution effects. Separate grids for ECAL and HCAL have been designed for both the FCC-hh and the HE-LHC detectors in order to accurately model the angular resolution on reconstructed jets. The FCC-hh detector features an improved angular resolution by a factor 2 in the ECAL and a factor 4 in the HCAL compared to the HE-LHC detector. The energy resolution of the calorimeters is assumed to be the same for both detectors and the calorimeter parameters are summarised in Table 2.

In DELPHES the information provided by the tracker and calorimeters is combined within the particle-flow algorithm for an optimal event reconstruction. If the momentum resolution of the tracking system is better than the energy resolution of calorimeters (typically for momenta below some threshold) the charged particles momenta are measured mainly through tracking. Vice-versa at high energy, calorimeters provide a better momentum measurement. The particle-flow algorithm exploits this complementarity to provide the best possible single charged particle measurement - the particle-flow tracks; these contain electron, muons and charged hadrons. Jet collections are then formed using several different input objects such as tracks (Track-jets), calorimeter (Calo-jets) and particle-flow candidates $(P F$ -
Fig. 1 Left: track reconstruction efficiency inside highly boosted QCD jets as function of the angular distance $\Delta R$ between the track and the center of the jet for different assumptions on the tracker spatial resolution. Right: reconstructed "soft-dropped" jet mass of highly boosted top and QCD jets with various sets of input to the jet clustering algorithm: tracks only, calorimeters towers only and particle-flow candidates
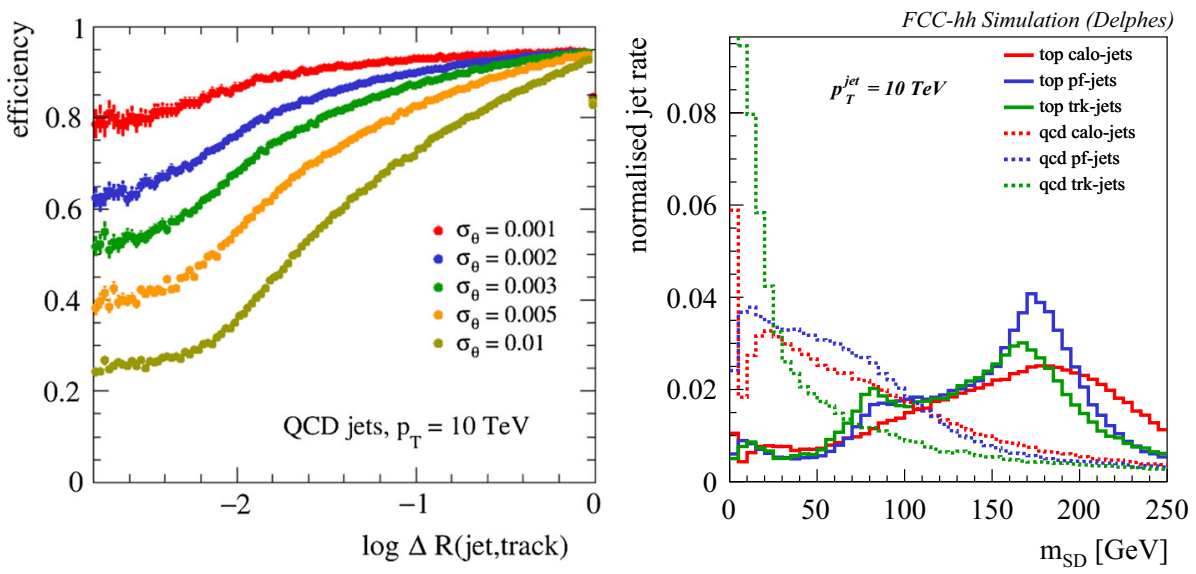
Table 2 Calorimeter parameters for the FCC-hh and HE-LHC detectors in Delphes

\begin{tabular}{lll}
\hline & FCC-hh & HE-LHC \\
\hline$\sigma(E) / E(\mathrm{ECAL})$ & $10 \% / \sqrt{E} \oplus 1 \%$ & $10 \% / \sqrt{E} \oplus 1 \%$ \\
$\sigma(E) / E(\mathrm{HCAL})$ & $50 \% / \sqrt{E} \oplus 3 \%$ & $50 \% / \sqrt{E} \oplus 3 \%$ \\
$\eta \times \phi$ cell size (ECAL) & $(0.01 \times 0.01)$ & $(0.02 \times 0.02)$ \\
$\eta \times \phi$ cell size (HCAL) & $(0.025 \times 0.025)$ & $(0.1 \times 0.1)$ \\
\hline
\end{tabular}

jets). The DELPHES framework integrates the FastJet package [53], allowing for jet reconstruction with the most popular jet clustering algorithms. In the present study the anti- $k_{T}$ algorithm [54] is used with several jet clustering $\mathrm{R}$ parameters $(R=0.2,0.4,0.8,1.5)$.

Common jet shape observables used for jet substructure analysis such as $\mathrm{N}$-subjettiness [55] and the soft-dropped mass [56] are computed on-the-fly and stored in the output jet collections. As an illustration, the reconstructed soft-dropped mass in the FCC-hh detector for top and QCD jets with $p_{\mathrm{T}}=$ $10 \mathrm{TeV}$ and cone size $R=0.2$ is shown in Fig. 1(right). Thanks to the superior tracker segmentation, we find Trackjets to perform better in terms of QCD background rejection despite the slightly worse jet mass resolution. A recent study shows that the reconstruction of jet substructure variables for highly boosted objects will benefit from small cell sizes of the hadronic calorimeter which confirms the FCC baseline design [57].

\subsubsection{Object identification efficiencies}

Trigger, reconstruction and identification efficiencies are parametrised as function of the particle momentum in DELPHES. Given that these parameterisations depend on the detailed knowledge of the detector, we simply use a global parameterisation for each object.

For electron and muons, the isolation around a cone is computed as the sum of the full list of particle-flow candidates within a cone of radius $R$ excluding the particle under consideration. No selection on the isolation variable is applied during DELPHES processing since the optimal selection working point is analysis and object dependent. Electrons and muons originating from heavy resonances are highly boosted and populate the central rapidity region of the detector. For the purpose of this study, flat reconstruction identification efficiencies are assumed (see Table 3).

The identification of jets that result from $\tau$ decays or heavy flavour quarks - b or c quarks - typically involves the input from tracking information, such as vertex displacement or low-level detector input such as hit multiplicity $[58,59]$. Such information is not available as a default in DELPHES. Instead, a purely parametric approach based on MC genera- tor information is used. The probability to be identified as $b$ or $\tau$ depends on user-defined parameterizations (see Table 3 ). The behaviour of usual heavy flavour tagging algorithms in regimes of extreme boosts is yet unknown. We make the conservative assumption of vanishing efficiency as a function of the transverse momentum for both $\mathrm{b}$ and $\tau$-jets, as shown in Table 3. This choice is motivated by the fact that decay products originating from highly boosted $\mathrm{b}$ and $\tau$ decays will be extremely collimated and highly displaced, making their reconstruction difficult.

A mis-tagging efficiency, that is, the probability that a particle other than $\mathrm{b}$ or $\tau$ will be wrongly identified as $\mathrm{a} b$ or a $\tau$ has been included in the simulation and assumes a similar falling behaviour as a function of the jet momentum. For b-tagging, the mistag efficiency are parameterised separately for light-jets (uds-quarks) and c-jets. For $\tau$-tagging, we consider only mis-identification from QCD jets. Table 4 summarises the main values for the mis-tagging efficiency.

\subsection{Treatment of the Monte Carlo samples}

The modelling of the backgrounds in the high- $p_{\mathrm{T}}$ tagging regimes is a challenging task. The requirement of $b$ tagging in some MC samples can drastically reduce the available statistics. This shortage of events that pass the $b$-tagging cut in the signal regime, in conjunction with the large cross section of some of the backgrounds can lead to very spiky templates. To overcome this problem the tag rate function (TRF) method is used. By using the TRF method, no event is cut based on its $b$-tagging count, but instead all the events are weighted. This weight can be interpreted as the probability of the given event to contain the desired number of $b$ jets. To achieve this, the tagging efficiency (a function of $\eta, p_{\mathrm{T}}$ and true jet flavour) was used to calculate the event weight based on the kinematics and flavour of the jets found in each event. Despite the fact that very large amount of Monte Carlo statistics have been simulated in bins of $H_{T}$ and the usage of TRF to save events, there are still large statistical fluctuations from high weight events. In order to reduce this effect, and when large fluctuations are observed, the background spectrum is fitted. Further details on the TRF and fitting procedure are given in Appendix B and C, respectively.

\subsection{Statistical analysis}

Hypothesis testing is performed using a modified frequentist method based on a profile likelihood that takes into account the systematic uncertainties as nuisance parameters that are fitted to the expected Monte Carlo. The full shape information is used, with help from the sidebands to reduce the effect of systematic uncertainties in the signal region. The test statistics $q_{\mu}$ is defined as the profile log-likelihood ratio: 
Table 3 Global reconstruction efficiency of high $p_{\mathrm{T}}$ central objects for the HE-LHC and FCC-hh detectors in Delphes

\begin{tabular}{llllll}
\hline & Electrons $(\%)$ & Muons $(\%)$ & Photons $(\%)$ & b-jets & $\tau$-jets \\
\hline FCC-hh & 99 & 95 & 95 & $\left(1-p_{\mathrm{T}}[\mathrm{TeV}] / 15\right) \cdot 85 \%$ & $\left(1-p_{\mathrm{T}}[\mathrm{TeV}] / 30\right) \cdot 60 \%$ \\
HE-LHC & 95 & 95 & 95 & $\left(1-p_{\mathrm{T}}[\mathrm{TeV}] / 5\right) \cdot 75 \%$ & $\left(1-p_{\mathrm{T}}[\mathrm{TeV}] / 5\right) \cdot 60 \%$ \\
\hline
\end{tabular}

Table 4 Mis-identification efficiency of high $p_{\mathrm{T}}$ central heavy flavour jets for the HE-LHC and FCC detectors in Delphes

\begin{tabular}{llll}
\hline & Light $(\mathrm{b}-\mathrm{tag})$ & Charm $(\mathrm{b}-\mathrm{tag})$ & $\mathrm{QCD}(\tau$-tag $)$ \\
\hline FCC-hh & $\left(1-p_{\mathrm{T}}[\mathrm{TeV}] / 15\right) \cdot 1 \%$ & $\left(1-p_{\mathrm{T}}[\mathrm{TeV}] / 15\right) \cdot 5 \%$ & $\left(8 / 9-p_{\mathrm{T}}[\mathrm{TeV}] / 30\right) \cdot 1 \%$ \\
HE-LHC & $\left(1-p_{\mathrm{T}}[\mathrm{TeV}] / 5\right) \cdot 1 \%$ & $\left(1-p_{\mathrm{T}}[\mathrm{TeV}] / 5\right) \cdot 10 \%$ & $\left(1-p_{\mathrm{T}}[\mathrm{TeV}] / 5\right) \cdot 1 \%$ \\
\hline
\end{tabular}

$q_{\mu}=-2 \ln \left(\mathcal{L}\left(\mu, \hat{\hat{\theta}}_{\mu}\right) / \mathcal{L}(\hat{\mu}, \hat{\theta})\right)$, where $\hat{\mu}$ and $\hat{\theta}$ are the values of the parameters that maximise the likelihood function (with the constraint $0 \leq \hat{\mu} \leq \mu$ ), and $\hat{\hat{\theta}}_{\mu}$ are the values of the nuisance parameters that maximise the likelihood function for a given value of $\mu$. In the absence of any significant deviation from the background expectation, $q_{\mu}$ is used in the $\mathrm{CL}_{\mathrm{s}}$ method $[60,61]$ to set an upper limit on the signal production cross-section times branching ratio at the $95 \%$ CL. For a given signal scenario, values of the production cross section (parameterised by $\mu$ ) yielding $\mathrm{CL}_{\mathrm{s}}<0.05$, where $\mathrm{CL}_{\mathrm{s}}$ is computed using the asymptotic approximation [62], are excluded at $95 \% \mathrm{CL}$. For a $5 \sigma$ discovery, the quantity $1-\mathrm{CL}_{\mathrm{b}}$ must be smaller than $2.87 \cdot 10^{-7}[60]$ and is also computed using the asymptotic approximation.

\section{Studies at $100 \mathrm{TeV}$}

\subsection{Leptonic final states}

The decay products of heavy resonances are in the multi$\mathrm{TeV}$ regime and the capability to reconstruct their momentum imposes stringent requirements on the detector design. In particular, reconstructing the track curvature of multi-TeV muons requires excellent position resolution and a large lever arm. In this section, the expected sensitivity is presented for a $Z^{\prime} \rightarrow \ell \ell$ (where $\ell=e, \mu$ ) and $Z^{\prime} \rightarrow \tau \tau$ separately.

\subsubsection{The $e^{+} e^{-}$and $\mu^{+} \mu^{-}$final states}

Events are required to contain two isolated opposite-sign leptons with $p_{\mathrm{T}}>1 \mathrm{TeV},|\eta|<4$ and an invariant mass $m_{l l}>$ $2.5 \mathrm{TeV}$. Figure 2 left shows the invariant mass for a $30 \mathrm{TeV}$ $Z_{\text {SSM }}^{\prime}$ signal for the $\mu \mu$ channel for FCC-hh. The di-electron invariant mass spectrum is not shown, but as expected from the calorimeter constant term that dominates the resolution at high $p_{\mathrm{T}}$, the mass resolution is better for the $e e$ channel. The di-lepton invariant mass spectrum is used as the discriminant and a $50 \%$ normalisation uncertainty on the background is assumed (this uncertainty is extremely conservative, but does not affect the final results, due to the negligible background in the largest mass regions). Figure 2 (right) shows the $95 \%$ $\mathrm{CL}$ exclusion limit obtained with $30 \mathrm{ab}^{-1}$ of data combining ee and $\mu \mu$ channels. Figure 4 (left) shows the integrated luminosity required to reach a $5 \sigma$ discovery as a function of the mass of the heavy resonance. The $Z^{\prime} \rightarrow$ ee and $Z^{\prime} \rightarrow \mu \mu$ channels display very similar performance due to the low background rates. We conclude therefore that the reference detector design features near to optimal performance for searches involving high $p_{\mathrm{T}}$ muon final states. Combining $e e$ and $\mu \mu$ channels, masses up to $\sim 41 \mathrm{TeV}$ can be excluded or discovered. The slope of the $5 \sigma$ discovery reach becomes softer after $15 \mathrm{TeV}$ because the search becomes almost free of backgrounds, which also explains why the $5 \sigma$ discovery reach is slightly better than the respective $95 \%$ CL exclusion limits.

\subsubsection{The $\tau^{+} \tau^{-}$final state}

At the LHC, the most sensitive channel to search for highmass di- $\tau$ resonances is when both $\tau$ leptons decay hadronically [63]. The analysis presented in this section focuses on this decay channel alone. The event selection requires two jets with $p_{T}>0.5 \mathrm{TeV}$ and $|\eta|<2.5$, both identified as $\tau$ 's. To ensure no overlap between the $\ell=e, \mu$ and $\tau$ final states, jets containing an electron or a muon with $p_{\mathrm{T}}>$ $100 \mathrm{GeV}$ are vetoed. The requirements of $\Delta \phi\left(\tau_{1}, \tau_{2}\right)>2$ and $2.5<\Delta R\left(\tau_{1}, \tau_{2}\right)<4$ are applied to suppress multi-jet backgrounds. Furthermore, mass dependent cuts are applied to maximise the signal significance and are summarised in Table 5. Several proxies for the true resonance mass have been tested, such as the invariant mass of the two $\tau$ 's, with and without correction for the missing energy, however the transverse mass ${ }^{2}$ provides the best sensitivity and is therefore used to estimate the sensitivity. Figure 3 shows the di- $\tau$ transverse mass (left) for a $10 \mathrm{TeV} Z_{\text {SSM }}^{\prime}$ and the $95 \%$ CL exclusion limits for $30 \mathrm{ab}^{-1}$ of data (right). The required integrated

${ }^{2}$ The transverse mass is defined as $m_{T}=$
$\sqrt{2 p_{\mathrm{T}}^{Z^{\prime}} * E_{T}^{\text {miss }} *\left(1-\cos \Delta \phi\left(Z^{\prime}, E_{T}^{\text {miss }}\right)\right)}$. 

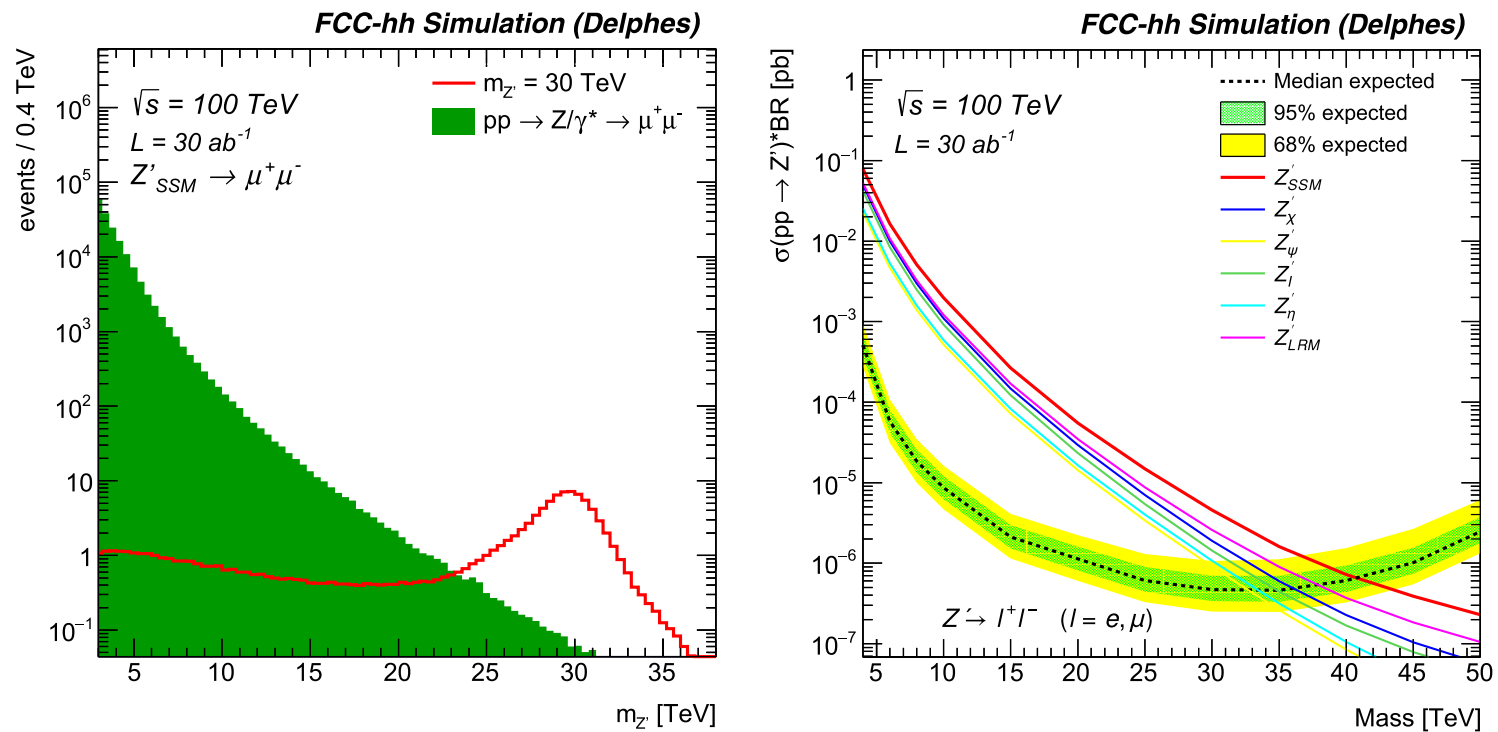

Fig. 2 Left: invariant mass for a $30 \mathrm{TeV}$ signal after full event selection for the $\mu \mu$ channel. Right: 95\% CL limit versus mass for the combined di-lepton $(e e, \mu \mu)$ channel

Table 5 List of mass dependent cuts optimised to maximise the sensitivity for the $Z^{\prime} \rightarrow \tau \tau$ search

\begin{tabular}{llll}
\hline$Z^{\prime}$ mass $[\mathrm{TeV}]$ & $\Delta \phi\left(\tau_{1}, \tau_{2}\right)$ & $\Delta R\left(\tau_{1}, \tau_{2}\right)$ & $E_{T}^{\text {miss }}$ \\
\hline $4-8$ & $>2.4$ & $>2.5$ and $<3.5$ & $>400 \mathrm{GeV}$ \\
10 & $>2.4$ & $>2.7$ and $<4$ & $>300 \mathrm{GeV}$ \\
$12-14$ & $>2.6$ & $>2.7$ and $<4$ & $>300 \mathrm{GeV}$ \\
$16-18$ & $>2.7$ & $>2.7$ and $<4$ & $>300 \mathrm{GeV}$ \\
$>18$ & $>2.8$ & $>3$ and $<4$ & $>300 \mathrm{GeV}$ \\
\hline
\end{tabular}

luminosity versus mass of the resonance to reach a $5 \sigma$ discovery is shown in Fig. 4 (right). The minimum of the curves around $6 \mathrm{TeV}$ is caused by the analysis not being optimised for the lowest mass points.

Heavy resonances decaying to $\tau$ leptons reconstructed in the hadronic decay mode are more challenging than $e e$ or $\mu \mu$, given the overwhelming multi-jet background. We find that masses up to $18 \mathrm{TeV}$ can be probed at the FCC-hh. We note that the assumed $\tau$ identification efficiency considered in this analysis is assumed to be conservative (see Sect. 3.2.3), and only a study based on full detector simulation could provide more realistic numbers.

\subsubsection{Sensitivity to flavour-anomaly inspired $Z^{\prime}$ models}

Recent measurements in $B \rightarrow K^{*} \mu^{+} \mu^{-}$decays from $\mathrm{LHCb}$ have revealed some tensions with the SM predictions [22,23]. This possibly indicates the existence of new physics at an energy scale that would be accessible to direct discovery. The reach for the "naive" flavour anomaly $Z^{\prime}$ model from Ref. [25] is studied in this section. In this model it is assumed that the $Z^{\prime}$ only couples to $b / s$ quarks $\left(g_{s b}\right)$ and to muons $\left(g_{\mu \mu}\right)$. This assumption is maximally conservative in the sense that it assumes a minimal set of non-vanishing couplings of the new resonance to quarks. Additional quark couplings would have the effect of increasing the production cross section thereby increasing the reach. For each $Z^{\prime}$ mass hypothesis $m_{Z^{\prime}}$, the product of $g_{s b}$ and $g_{\mu \mu}$ is fixed by the observed $R_{K^{(*)}}$ anomalies:

$\frac{g_{b s} g_{\mu \mu}}{m_{Z^{\prime}}^{2}} \approx \frac{1}{(30 \mathrm{TeV})^{2}}$.

In this study only one direction in the $\left(g_{s b}, g_{\mu \mu}\right)$ plane $^{3}$ has been explored in which both couplings are re-scaled by a common mass dependent factor compatible with Eq. 4.1. The scaling of the couplings is defined by: $g_{\mu \mu}=m_{Z^{\prime}} /(5 \mathrm{TeV})$ and $g_{s b}=0.023 m_{Z^{\prime}} /(5 \mathrm{TeV})$.

The event selection strategy described in Sect. 4.1.1 has been applied here. Figure 5 shows the invariant mass of the $\mu^{+} \mu^{-}$system (left) for $m_{Z^{\prime}}=10 \mathrm{TeV}$, the $95 \%$ CL exclusion limit obtained (right) and the integrated luminosity required to reach a $5 \sigma$ discovery as a function of the mass of the $\mu^{+} \mu^{-}$ system (bottom). We find that $m_{Z^{\prime}}<25 \mathrm{TeV}$ can be excluded with $\mathcal{L}=30 \mathrm{ab}^{-1}$. A $6 \mathrm{TeV}$ mass could be discovered with less integrated luminosity than a $4 \mathrm{TeV}$ mass because for this particular model the signal rate increases slower than the background. Models with $m_{Z^{\prime}}>18 \mathrm{TeV}$ are not allowed because they either violate unitarity or they are incompatible with $B_{S}-\bar{B}_{S}$ mixing measurements [64]. We conclude therefore that the full allowed mass range can be excluded

\footnotetext{
3 More precisely, constraints from $B_{s}-\bar{B}_{s}$ mixing measurements and from unitarity set an upper limit on the allowed $g_{s b}$ and $g_{\mu \mu}$ parameters.
} 

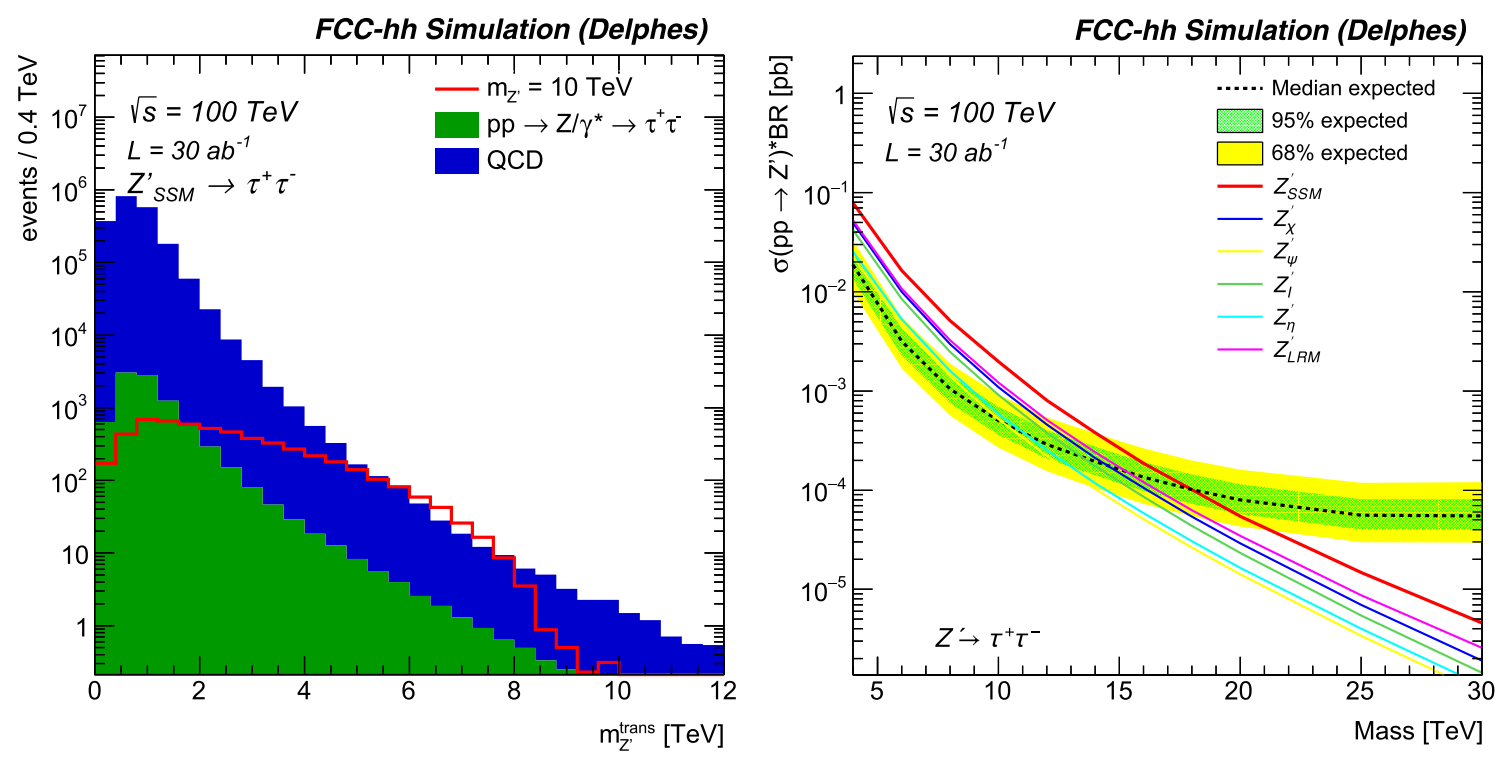

Fig. 3 Left: Di- $\tau$ transverse mass for a $10 \mathrm{TeV}$ signal after full event selection. Right: 95\% CL limit versus mass
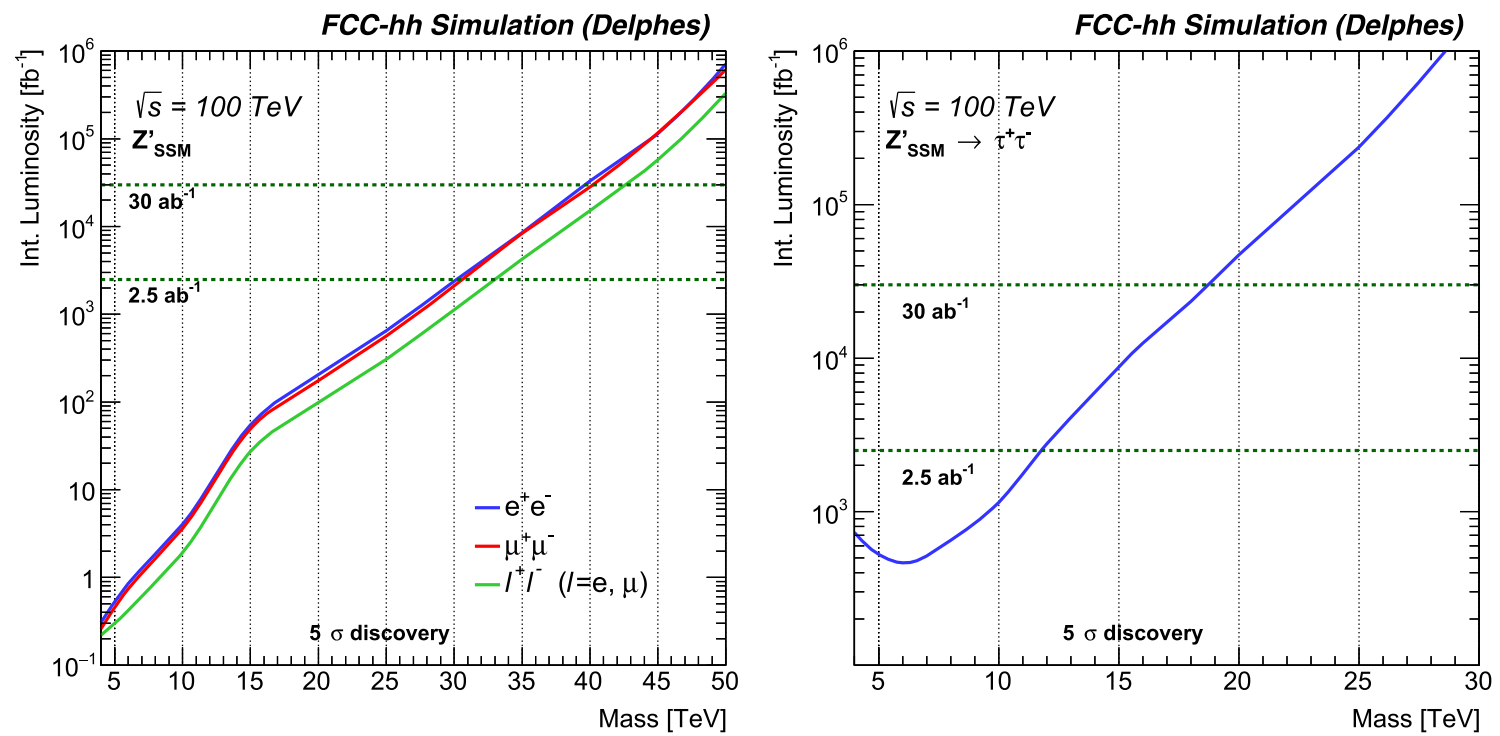

Fig. 4 Integrated luminosity versus mass for a $5 \sigma$ discovery, for $e / \mu$ final states (left) and for the $\tau$ final states (right)

at the FCC-hh with the detector performance discussed in Sect. 3.2, in agreement with the findings in Ref. [25]. We note that further studies involving more realistic models can be found in Ref. [26].

\subsection{Hadronic final states}

Heavy resonances decaying hadronically also impose stringent requirements on the detector design. Precise jet energy resolution requires full longitudinal shower containment. Highly boosted top quarks and $W$ bosons decay into highly collimated jets and differ from standard QCD jets by a characteristic inner jet sub-structure. An excellent granularity both in the tracking detectors and calorimeters is therefore required to resolve the sub-structure of jets since a high discrimination power against QCD backgrounds can result in increased sensitivity in heavy resonance searches.

\subsubsection{Multi-variate object tagging}

An important ingredient of the hadronic searches is the identification of heavy boosted top quarks and $W$ bosons. Two object-level taggers using boosted decision trees (BDT) were developed to discriminate $W$ and top jets against the light jet flavours treated as background. Top and $W$ taggers were optimised using jets with a transverse boost of $p_{\mathrm{T}}=10 \mathrm{TeV}$. At 

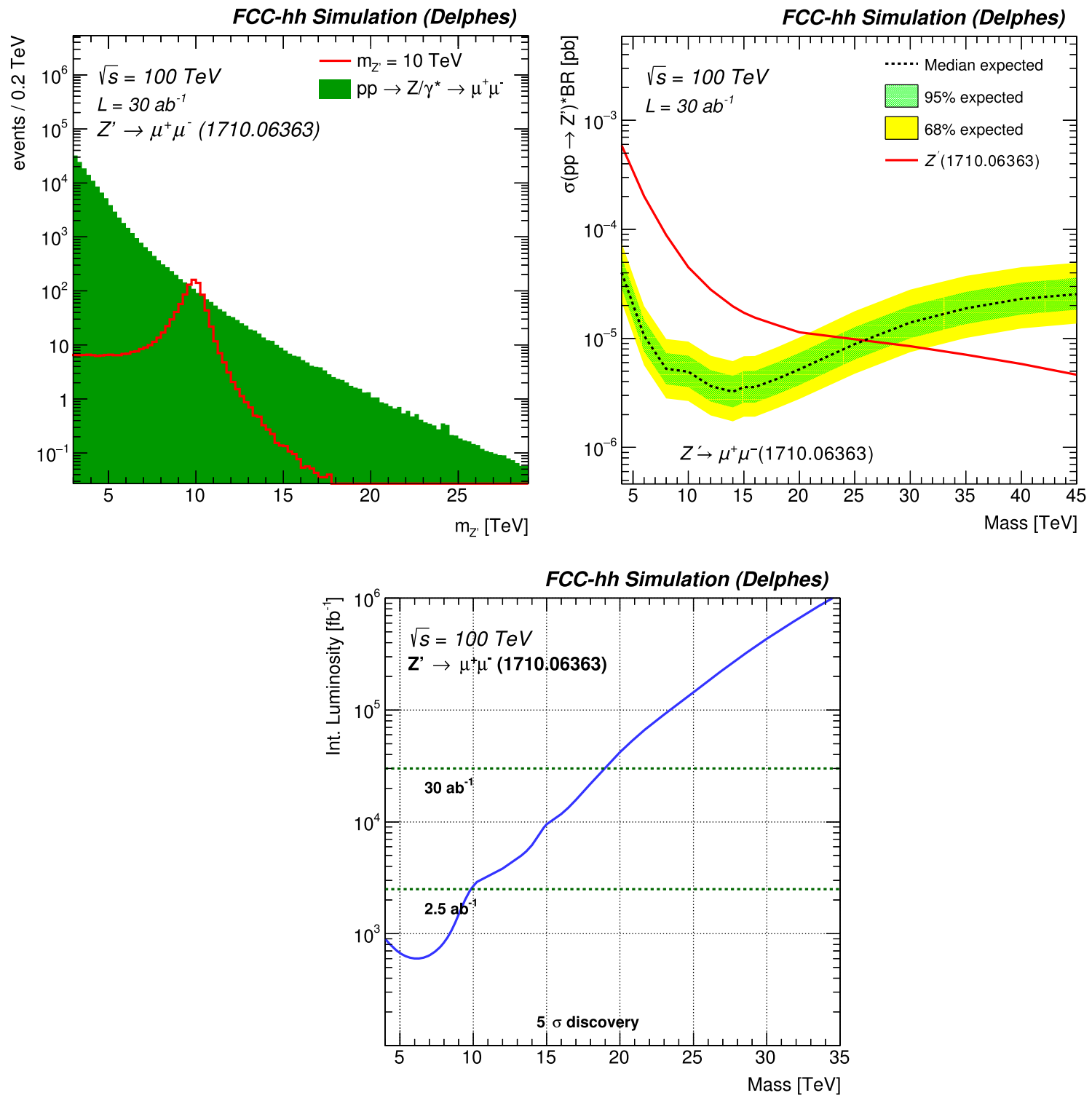

Fig. 5 Left: invariant mass for a $10 \mathrm{TeV}$ signal after full event selection in flavour anomaly scenario. Limit versus mass (right) and luminosity for a $5 \sigma$ discovery (bottom)

these extreme energies, $W$ and top jets have a characteristic angular size $R=0.01-0.02$, i.e., smaller than the typical electromagnetic and hadronic calorimeter cells. Following the approach described in [65], we exploit the superior track angular resolution and reconstruct jets from tracks only using the anti- $k_{T}$ algorithm with a parameter $\mathrm{R}=0.2$, but also larger values are used to increase the discrimination power of the BDT. The missing neutral energy is corrected for by rescaling the track 4-momenta by the factor $p_{\mathrm{T}, t r k} / p_{\mathrm{T}, P F}$, where $p_{\mathrm{T}, t r k}$ is the track jet $p_{\mathrm{T}}$ and $p_{\mathrm{T}, P F}$ is the particle-flow (PF) Jet $p_{\mathrm{T}}$. In what follows, we will simply refer to "track jets" as the jet collection that includes the aforementioned rescaling. The boosted top tagger is built from jet substructure observables: the soft-dropped jet mass [56] $\left(m_{S D}\right)$ and
$\mathrm{N}$-subjettiness [55] variables $\tau_{1,2,3}$ and their ratios $\tau_{2} / \tau_{1}\left(\tau_{21}\right)$ and $\tau_{3} / \tau_{2}\left(\tau_{32}\right)$. The $W$-jet tagger also uses "isolation-like" variables, first introduced in Ref. [66] that exploit the absence of high $p_{\mathrm{T}}$ final-state radiation (FSR) in the vicinity of the $W$ decay products. We call these variables $E_{F}(n, \alpha)$ and define them as:

$$
E_{F}(n, \alpha)=\sum_{\frac{n-1}{5} \alpha<\Delta R(k, j e t)<\frac{n}{5} \alpha} p_{\mathrm{T}}^{(k)} / \sum_{\Delta R(k, j e t)<\alpha} p_{\mathrm{T}}^{(k)},
$$

with $k$ running over the jet constituents and $\alpha=0.05$. We construct five variables $E_{F}(n, \alpha)$ with $n=[1,2,3,4,5]$ and use them as input to the BDT. The $W$ tagger has signif- 

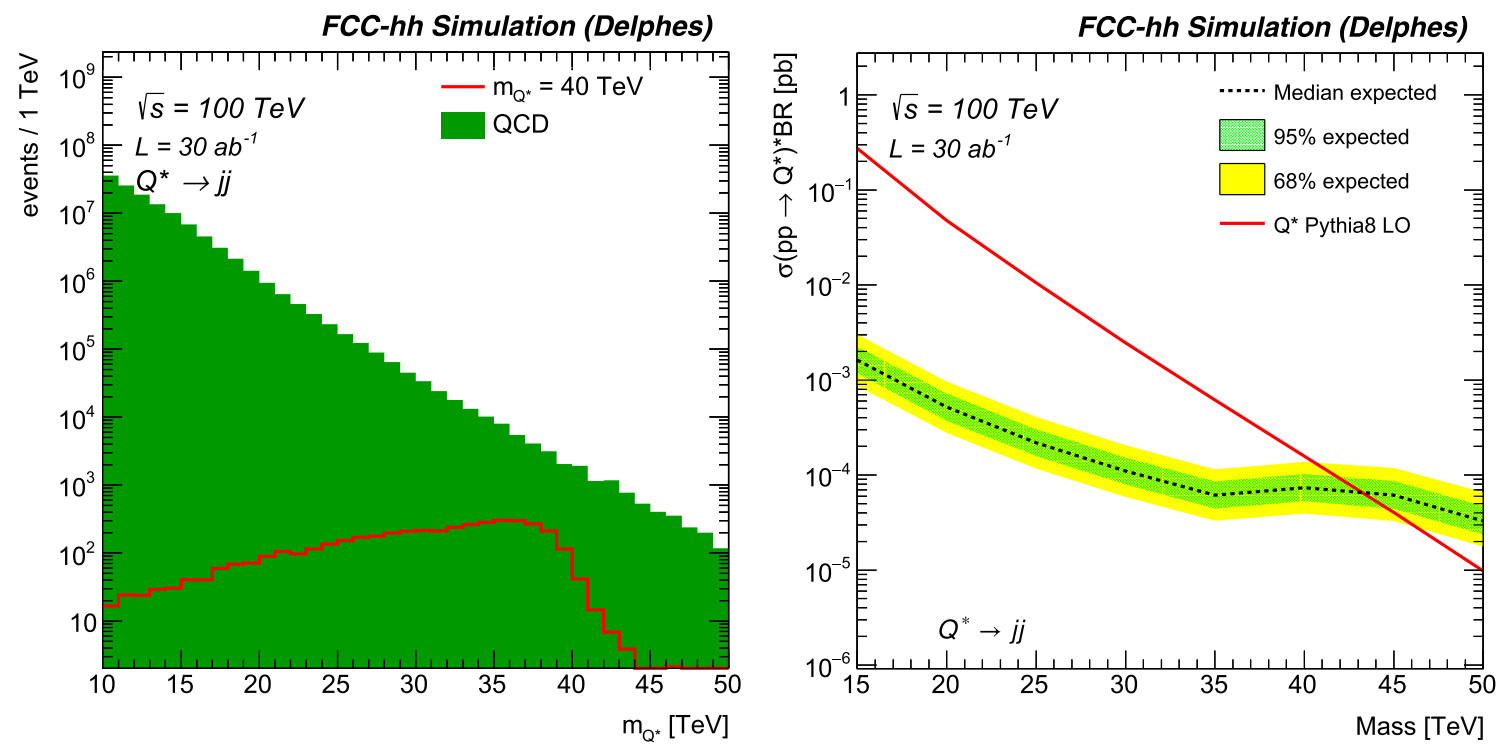

Fig. 6 Invariant mass distribution of the two selected jets for a $40 \mathrm{TeV}$ signal (left), 95\% CL limit versus mass (right)

icantly better performance than the top tagger thanks to the excellent discrimination power of the energy-flow variables. We choose the working points for the analyses presented later, with a top and $W$ tagging efficiencies of $\epsilon_{S}^{\text {top }}=60 \%$ and $\epsilon_{S}^{\mathrm{W}}=90 \%$, corresponding to a background rejection of $\epsilon_{B}^{\text {top }}=\epsilon_{B}^{\mathrm{W}}=90 \%$. More details on the multi-Variate object tagging can be found in Appendix D.

\subsubsection{The jj final state}

Jets are clustered using particle-flow candidates with the anti$k_{T}$ [54] algorithm and parameter $\mathrm{R}=0.4$. We require at least two very energetic jets with $p_{\mathrm{T}}>3 \mathrm{TeV}$ and $|\eta|<3$. As dijet events from the signal will tend to be more central than for the background, the rapidity difference between the two leading jets $\Delta\left(\eta_{1}, \eta_{2}\right)$ is required to be smaller than 1.5 . The di-jet invariant mass for the $Q^{*} \rightarrow j j$ signal with a mass of $40 \mathrm{TeV}$ together with the QCD contribution after the full event selection, is shown on Fig. 6 (left). The right figure shows the $95 \%$ CL exclusion limit obtained with $30 \mathrm{ab}^{-1}$ of data, and the left panel of Fig. 8 shows the integrated luminosity required to reach a $5 \sigma$ discovery as a function of the $\mathrm{Q}^{*}$ mass. For this very simple case of a strongly coupled object we reach $95 \% \mathrm{CL}$ exclusion limits of $43 \mathrm{TeV}$ and $5 \sigma$ discovery reach of $40 \mathrm{TeV}$ with $30 \mathrm{ab}^{-1}$ of integrated luminosity.

\subsubsection{The tī final state}

To resolve the jet sub-structure, track jets are found to perform better compared to particle-flow jets, thus the $G_{R S} \rightarrow$ $W^{+} W^{-}$and $Z^{\prime} \rightarrow t \bar{t}$ searches make use of track jets. As no lepton veto is applied, there is also some acceptance for leptonic decays and the sensitivity to semi-leptonic or $t \bar{t}$ decays is enhanced by adding the $\mathbf{p}_{\mathrm{T}}{ }^{\text {miss }}$ vector to the closest jet 4-momentum (among the two leading jets). We require two jets with a $p_{\mathrm{T}}>3 \mathrm{TeV}$ and $|\eta|<3$ and $\Delta\left(\eta_{1}, \eta_{2}\right)<2.4$. Both jets must be tagged as "top jets" (using the tagger defined in Sect. 4.2.1). In addition, the two selected high- $p_{\mathrm{T}}$ jets must be tagged as b-jets. Finally, to further reject QCD events, we require for both jets the soft-dropped mass to be larger than $40 \mathrm{GeV}$. Figure 7 (left) shows the di-top invariant mass distribution after the final event selection for a $20 \mathrm{TeV}$ signal from a Topcolor $Z^{\prime}$ and backgrounds. Thanks to the BDT discriminant, the largest background contribution is top pair production itself and the QCD contribution is now the second leading one. The right panel shows the 95\% CL exclusion limit obtained with $30 \mathrm{ab}^{-1}$ of data and the right panel of Fig. 8 shows the integrated luminosity required to reach a $5 \sigma$ discovery as a function of the $\mathrm{Z}^{\prime}$ mass. Further developments to improve the mass resolution could be considered to improve the sensitivity, but already with such wide spectrum, exclusions between 24 and $28 \mathrm{TeV}$ and discoveries between 18 and $23 \mathrm{TeV}$ are reached depending on the model $\left(Z_{\text {SSM }}^{\prime}\right.$ or leptophobic $\left.Z_{\mathrm{TC} 2}^{\prime}\right)$. A more extensive study of the $t \bar{t}$ decay at a $100 \mathrm{TeV}$ collider ignoring the simulation of the detector response can be found in Ref. [67].

\subsubsection{The $W^{+} W^{-}$final state}

The event selection in this case consists of two jets with a $p_{\mathrm{T}}>3 \mathrm{TeV},|\eta|<3$ and $\Delta\left(\eta_{1}, \eta_{2}\right)<2.4$. Both jets must be $W$ tagged (see Sect. 4.2.1). Again, to further reject QCD events, we require for both jets the soft-dropped mass to be 

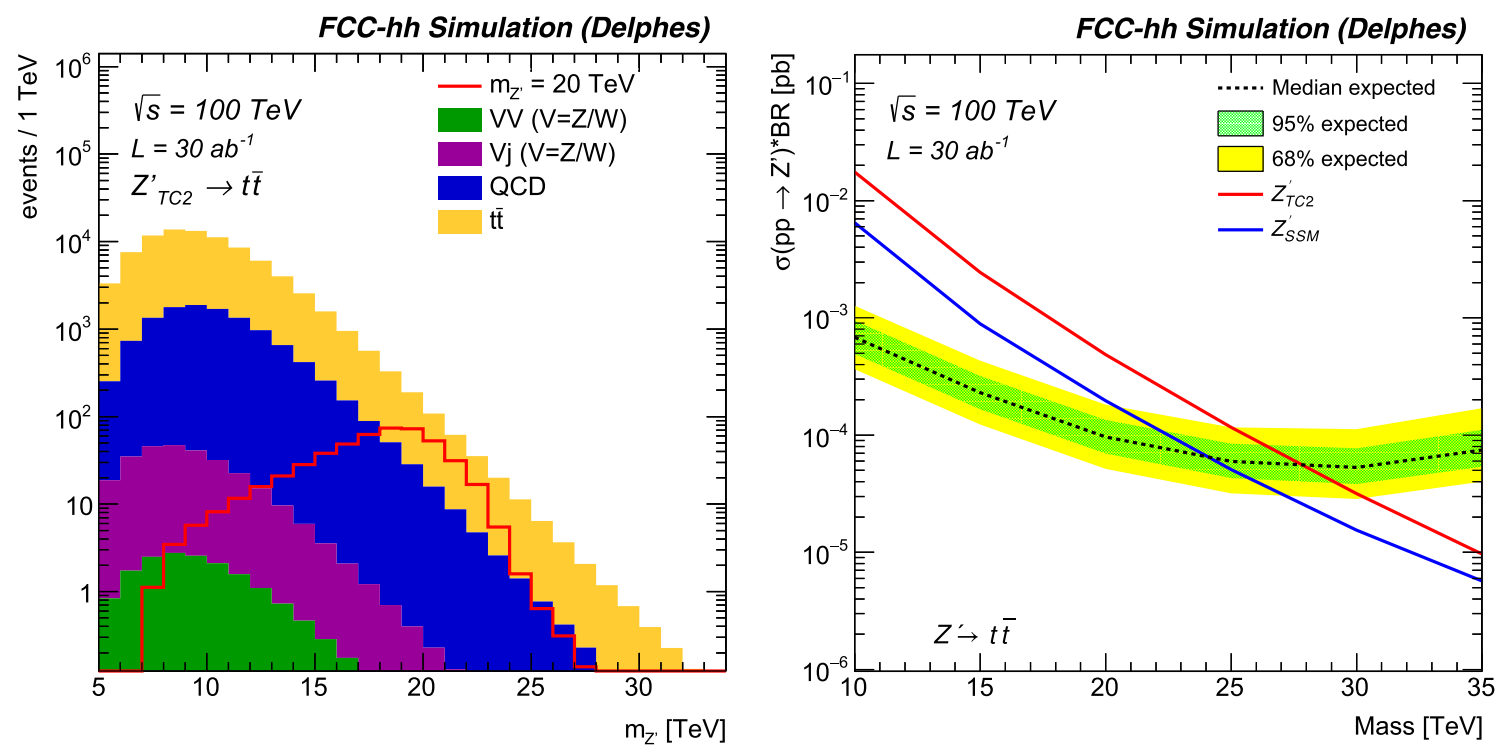

Fig. 7 Invariant mass distribution of the two selected top-jets (left) for a $20 \mathrm{TeV}$ signal (left), 95\% CL limit versus mass (right)
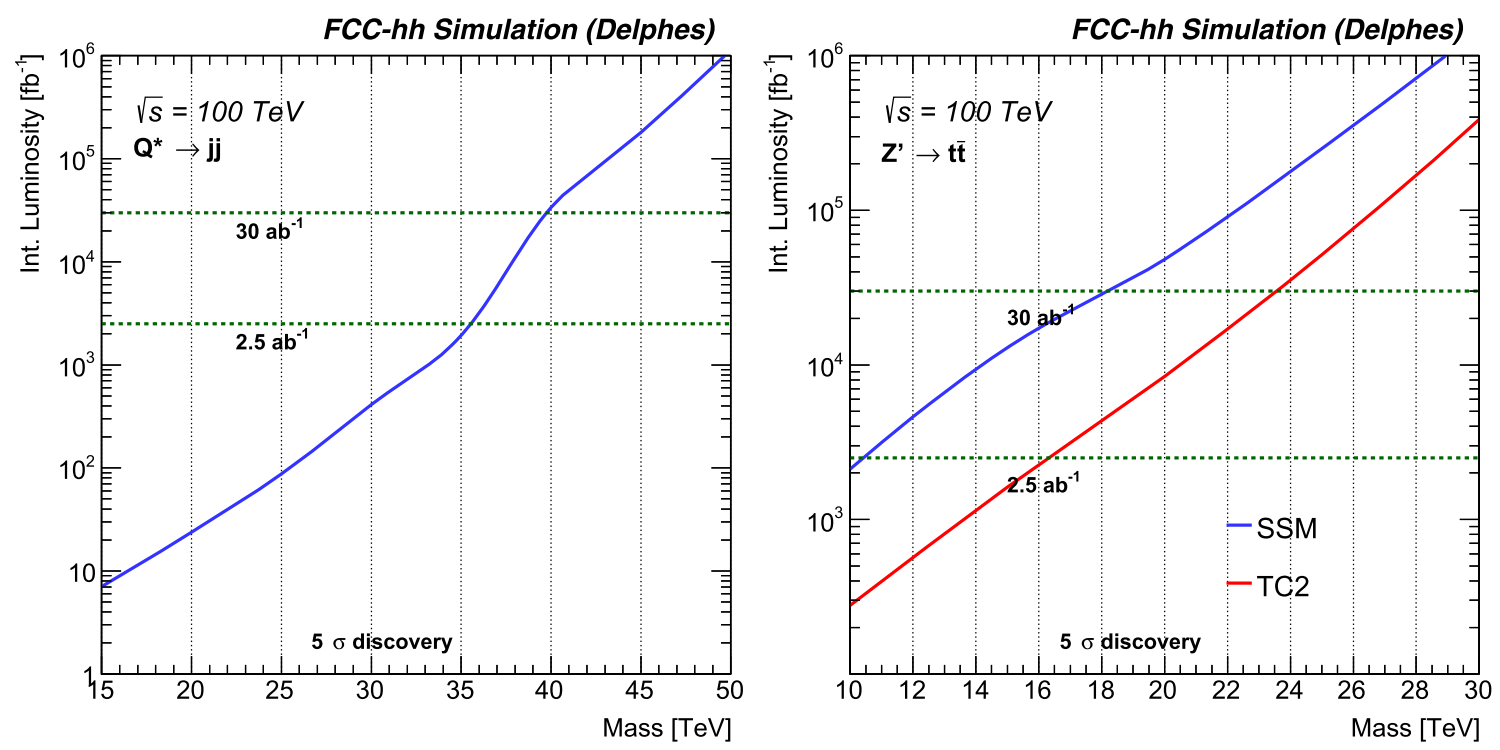

Fig. $85 \sigma$ discovery reach for the $j j$ (left) and $t \bar{t}$ (right) final states

larger than $40 \mathrm{GeV}$. Figure 9 (left) shows the di-boson invariant mass distribution after the final selection for a $20 \mathrm{TeV}$ signal and background. Given the very good performance of the BDT discriminant, the QCD contribution is greatly reduced. The right panel shows the 95\% CL exclusion limit obtained with $30 \mathrm{ab}^{-1}$ of data and the bottom panel shows the integrated luminosity required to reach a $5 \sigma$ discovery as a function of the Randall-Sundrum graviton mass. Further developments to improve the W-jet/QCD could be considered, to improve the sensitivity as well as combining with leptonic channels, but, already with the current assumptions, the exclusion of $28 \mathrm{TeV}$ (Fig. 9 right) and the discovery of a
$22 \mathrm{TeV}$ signal are obtained (Fig. 9 bottom) . Further studies in the context of composite Higgs models can be found in Ref. [68].

\section{Comparison with the $27 \mathrm{TeV}$ HE-LHC}

We briefly present here the results of our study for the $27 \mathrm{TeV}$ HE-LHC. The detector simulation is based on the hybrid ATLAS/CMS HL-LHC detector parameterisation introduced in Sect. 3.2. The analysis strategies remain identical to what was already presented in the previous sections, the only dif- 

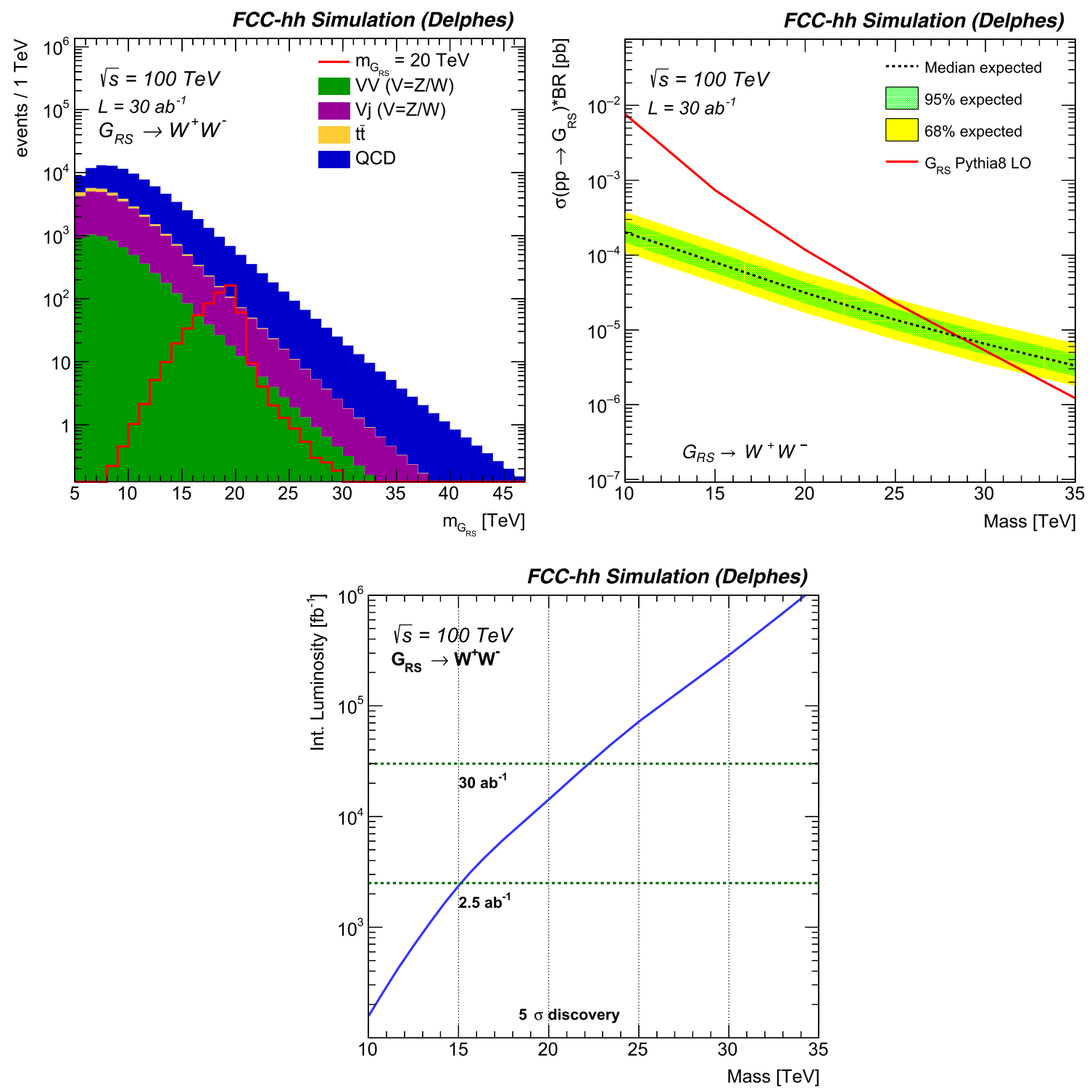

Fig. 9 Invariant mass distribution of the two selected $\mathrm{W}$-jets for a $20 \mathrm{TeV}$ signal (left), 95\% CL limit versus mass (right) and $5 \sigma$ discovery reach (bottom)

ference being the re-optimisation of some selection thresholds, to account for the lower center of mass energy. The changes can be summarised as follows:

- $Z^{\prime} \rightarrow$ ee and $Z^{\prime} \rightarrow \mu \mu$ : lepton $p_{\mathrm{T}}$ threshold lowered from 1 to $0.5 \mathrm{TeV}$.

- $Z^{\prime} \rightarrow \tau \tau$ : mass dependent selection as shown in Table 6 .

- $G_{R S} \rightarrow W^{+} W^{-}, Z^{\prime} \rightarrow t \bar{t}, Q^{*} \rightarrow j j:$ jet $p_{\mathrm{T}}$ threshold lowered from 3 to $1 \mathrm{TeV}$.

The results are summarised in Fig. 10, together with a comparison to FCC-hh for the 95\% CL (left) and $5 \sigma$ discovery reach (right). Additional summary plots for FCC-hh and HELHC alone can be found in Appendix E. A more extensive
Table 6 Mass dependent cuts optimised to maximise the sensitivity for the $Z^{\prime} \rightarrow \tau \tau$ resonance search

\begin{tabular}{llll}
\hline$Z^{\prime}$ mass $[\mathrm{TeV}]$ & $\Delta \phi\left(\tau_{1}, \tau_{2}\right)$ & $\Delta R\left(\tau_{1}, \tau_{2}\right)$ & $E_{T}^{\text {miss }}$ \\
\hline 2 & $>2.4$ & $>2.4$ and $<3.9$ & $>80 \mathrm{GeV}$ \\
4 & $>2.4$ & $>2.7$ and $<4.4$ & $>80 \mathrm{GeV}$ \\
6 & $>2.4$ & $>2.9$ and $<4.4$ & $>80 \mathrm{GeV}$ \\
8 & $>2.6$ & $>2.9$ and $<4.6$ & $>80 \mathrm{GeV}$ \\
10 & $>2.8$ & $>2.9$ and $<4.1$ & $>60 \mathrm{GeV}$ \\
\hline
\end{tabular}

study of the di-jet decay at the HE-LHC ignoring the simulation of the detector response can be found in Ref. [69]. 

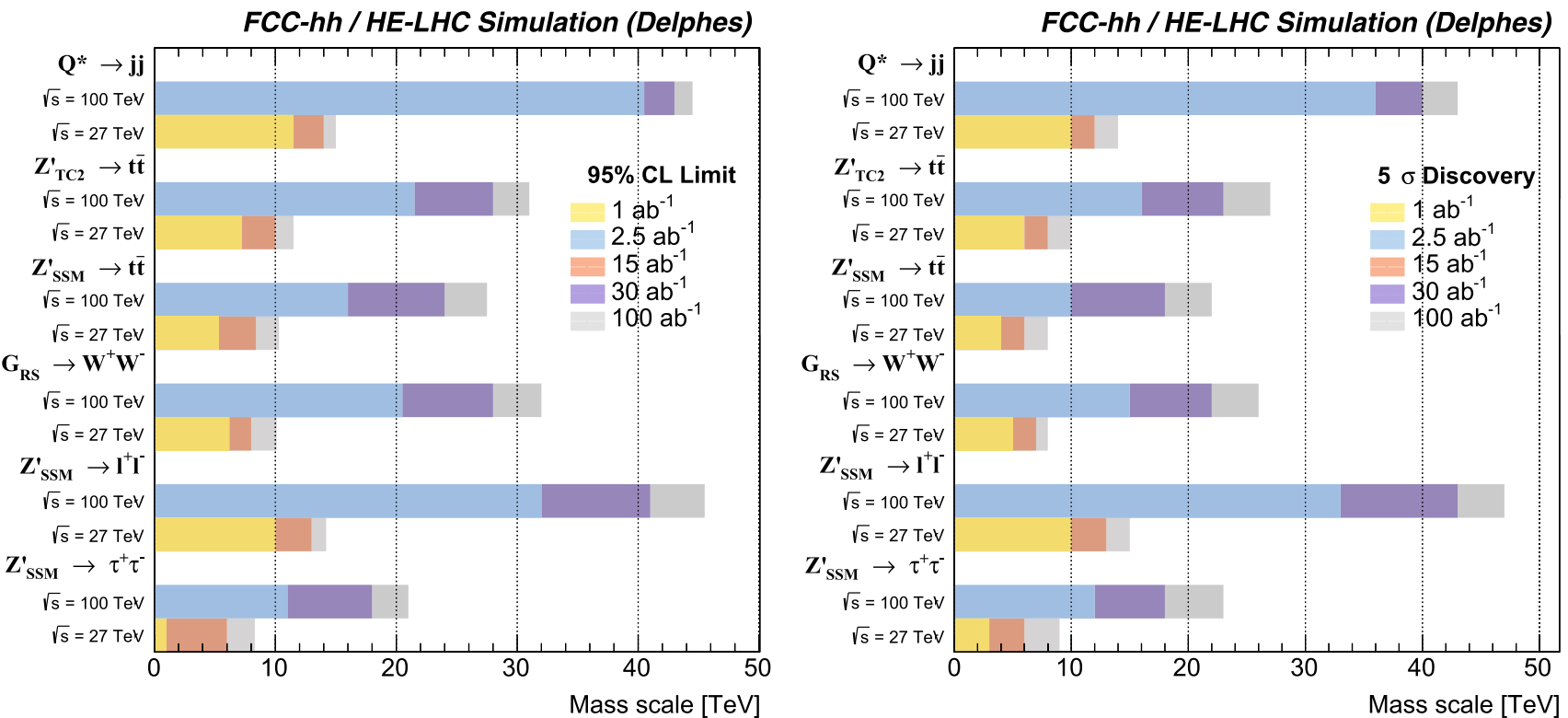

Fig. 10 Summary of the $95 \%$ CL limits (left) and $5 \sigma$ discovery reach (right) as a function of the resonance mass for different luminosity scenarios of FCC-hh and HE-LHC

\section{Characterisation of a $Z^{\prime}$ discovery}

\subsection{Context of the study}

We consider in this section a scenario in which a heavy dilepton resonance is observed by the end of HL-LHC run. In this case, considering that current limits are already pushing to quite high values the possible mass range, a collider with higher energy in the , would be needed to study the resonance properties, since too few events will be available at $\sqrt{s}=14 \mathrm{TeV}$. In this section we present the discrimination potential, among six $Z^{\prime}$ models, of the $27 \mathrm{TeV}$ HE-LHC, with an assumed integrated luminosity of $\mathcal{L}=15 \mathrm{ab}^{-1}$. Under the assumption that these $Z^{\prime}$ 's decay only to SM particles, we show that there are sufficient observables to perform this model differentiation in most cases.

\subsection{Bounds from HL-LHC}

As a starting point we need to estimate what are, for $\sqrt{s}=$ $14 \mathrm{TeV}$, the typical exclusion/discovery reaches for standard reference $Z^{\prime}$ models, assuming $\mathcal{L}=3 \mathrm{ab}^{-1}$ and employing only the $e^{+} e^{-}$and $\mu^{+} \mu^{-}$channels. To address this and the other questions below we will use the same set of $Z^{\prime}$ models as employed in Ref. [70] and mostly in Ref. [71]. We employ the MMHT2014 NNLO PDF set [72] throughout, with an appropriate constant $K$-factor (= 1.27) to account for higher order QCD corrections. The production cross section times leptonic branching fraction is shown in Fig. 11 (left) for these models at $\sqrt{s}=14 \mathrm{TeV}$ in the narrow width approximation
(NWA). We assume here that these $Z^{\prime}$ states only decay to SM particles.

Using the present ATLAS and CMS results at $13 \mathrm{TeV}$, [73] and [74], it is straightforward to estimate by extrapolation the exclusion reach at $\sqrt{s}=14 \mathrm{TeV}$ using the combined $e e+\mu \mu$ final states. This is given in the first column of Table 7. For discovery, only the $e e$ channel is used, due to the poor $\mu \mu$ pair invariant mass resolution near $M_{Z^{\prime}}=6 \mathrm{TeV}$. Estimates of the $3 \sigma$ evidence and $5 \sigma$ discovery limits are also given in Table 7. This naive extrapolation can be compared to the ATLAS HL-LHC prospect analysis in Ref. [1] and is found to be agreement. Based on these results, we will assume in our study for the HE-LHC that we are dealing with a $Z^{\prime}$ of mass $6 \mathrm{TeV}$. Figure 11 (right) shows the NWA cross sections for the same set of models, at $\sqrt{s}=27 \mathrm{TeV}$. We note that very large statistical samples will be available, with $\mathcal{L}=15 \mathrm{ab}^{-1}$, for $M_{Z^{\prime}}=6 \mathrm{TeV}$ and in both dilepton channels.

\subsection{Definition of the discriminating variables}

The various $Z^{\prime}$ models can be disentangled with the help of 3 inclusive observables: the production cross sections for different leptonic and hadronic final states, the leptonic forwardbackward asymmetry $A_{F B}$ and the rapidity ratio $r_{y}$. The variable $A_{F B}$ can be seen as an estimate of the charge asymmetry

$A_{F B}=A_{C}=\frac{\sigma(\Delta|y|>0)-\sigma(\Delta|y|<0)}{\sigma(\Delta|y|>0)+\sigma(\Delta|y|<0)}$,

where $\Delta|y|=\left|y_{l}\right|-\left|y_{\bar{l}}\right|$. This definition is equivalent to

$A_{F B}=\frac{\sigma_{F}-\sigma_{B}}{\sigma_{F}+\sigma_{B}}$, 

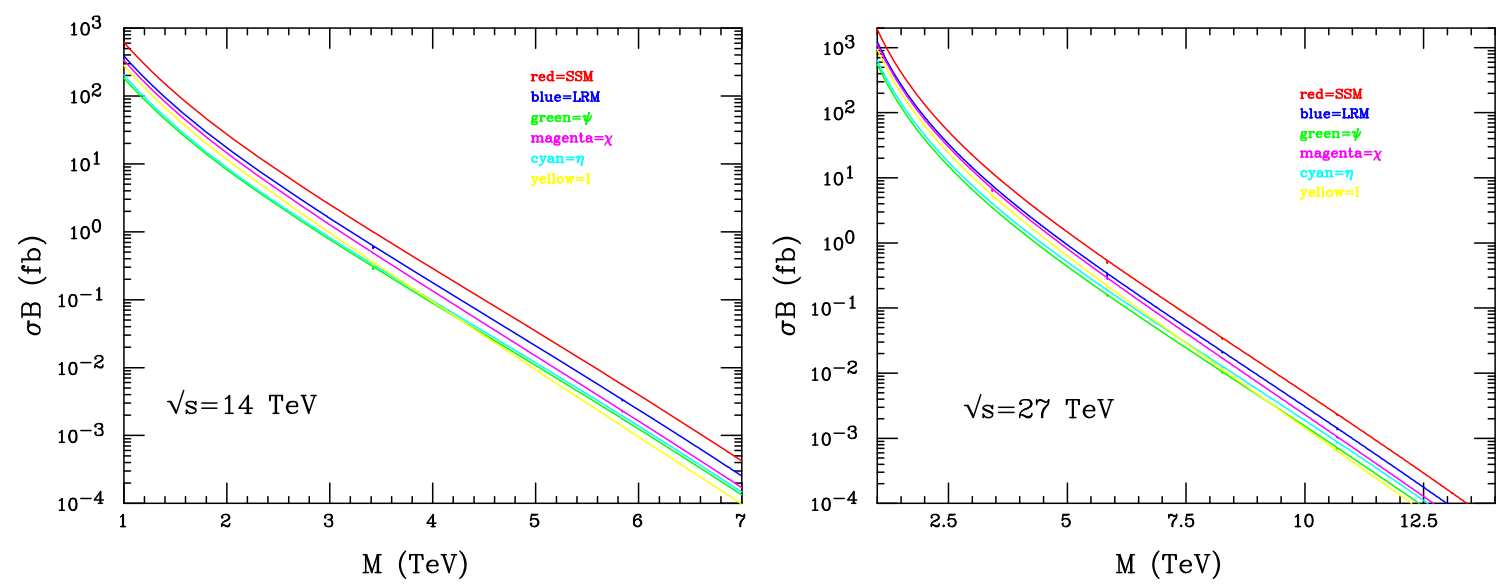

Fig. 11 Left: $\sigma B_{l}$ in the NWA for the $Z^{\prime}$ production at the $\sqrt{s}=14 \mathrm{TeV}$ LHC as functions of the $Z^{\prime}$ mass: SSM(red), LRM (blue), $\psi$ (green), $\chi$ (magenta), $\eta$ (cyan), I(yellow). (Right) $\sigma B_{l}$ of $Z^{\prime}$ in models described in (left) at $\sqrt{s}=27 \mathrm{TeV}$

Table 7 Mass reach for several $Z^{\prime}$ models at $\sqrt{s}=14 \mathrm{TeV}$ with $\mathcal{L}=$ $3 \mathrm{ab}^{-1}$

\begin{tabular}{llll}
\hline Model & $95 \%$ C.L. & $3 \sigma$ & $5 \sigma$ \\
\hline SSM & 6.62 & 6.09 & 5.62 \\
LRM & 6.39 & 5.85 & 5.39 \\
$\psi$ & 6.10 & 5.55 & 5.07 \\
$\chi$ & 6.22 & 5.68 & 5.26 \\
$\eta$ & 6.15 & 5.59 & 5.16 \\
I & 5.98 & 5.45 & 5.05 \\
\hline
\end{tabular}

with $\sigma_{F}=\sigma\left(\cos \theta_{c S}^{*}\right)>0$ and $\sigma_{B}=\sigma\left(\cos \theta_{c S}^{*}\right)<0$ where $\theta_{c s}^{*}$ is the Collins-Soper frame angle. The variable $r_{y}$ is defined as the ratio of central over forward events:

$r_{y}=\frac{\sigma\left(\left|y_{Z^{\prime}}\right|<y_{1}\right)}{\sigma\left(y_{1}<\left|y_{Z^{\prime}}\right|<y_{2}\right)}$,

where $y_{1}=0.5$ and $y_{2}=2.5$.

The results in this section have been obtained assuming the DELPHES[43] parametrisation of the HE-LHC detector [45]. In such a detector, muons at $\eta \approx 0$ are assumed to be reconstructed with a resolution $\sigma(p) / p \approx 7 \%$ for $p_{\mathrm{T}}=3 \mathrm{TeV}$.

\subsubsection{Leptonic final states}

The potential for discriminating various $Z^{\prime}$ models is first investigated using the leptonic $e e$ and $\mu \mu$ final states only. The signal samples for the 6 models have been generated with PYTHIA8[40] as described in Sect. 3.1 with the only difference being that the interference between the signal and Drell-Yan is included. The $Z^{\prime}$ decays assume lepton flavour universality, with branching ratio $B_{l}$. For a description of the event selection and a discussion of the discovery potential in leptonic final states for the list of $Z^{\prime}$ models being discussed here, the reader should refer to Sects. 4.1 and 5. We simply point out here that with $\mathcal{L}=15 \mathrm{ab}^{-1}$, all $Z^{\prime}$ models with $m_{Z^{\prime}} \lesssim 10 \mathrm{TeV}$ can be excluded at $\sqrt{s}=27 \mathrm{TeV}$.

Figure 12 (left) shows the correlated predictions for the $A_{F B}$ and the rapidity ratio $r_{y}$ observables defined previously, for these six models given the above assumptions. Although the interference with the SM background was included in the simulation, its effect is unimportant due to the narrowness of the mass window around the resonance that was employed. Furthermore, the influence of the background uncertainty on the results has been found to have little to no impact on the model discrimination potential. Therefore the displayed errors on $A_{F B}$ and $r_{y}$ are of statistical origin only. The results show that apart from a possible near degeneracy in models $\psi$ and $\eta$, a reasonable $Z^{\prime}$ model separation can indeed be achieved.

Using a profile likelihood technique, the signal strength $\mu$, or equivalently, $\sigma \times B_{l}$, can be fitted together with its corresponding error using the di-lepton invariant mass shape. The quantity $\sigma \times B_{l}$ and its total estimated uncertainty is shown in Fig. 12 (right) as a function of the integrated luminosity. The $\sigma \times B_{l}$ measurement seems to be able to resolve the degeneracy between the $\psi$ and $\eta$ models with $\mathcal{L}=15 \mathrm{ab}^{-1}$. It should be noted however that since the cross-section can easily be modified by an overall rescaling of the couplings or via the existence of decays into non-SM states, further handles will be needed for a convincing discrimination.

\subsubsection{Hadronic final states}

Model discrimination can be improved by including an analysis involving three additional hadronic final states: $t \bar{t}, b \bar{b}$ and $q \bar{q}$, where $q=u, d, c, s$. The sample production and event selection for the $t \bar{t}, q \bar{q}$ final states have been described in Sect. 4.2. We simply remind the reader that the analysis involves requiring the presence of two central high- $p_{\mathrm{T}}$ jets. 

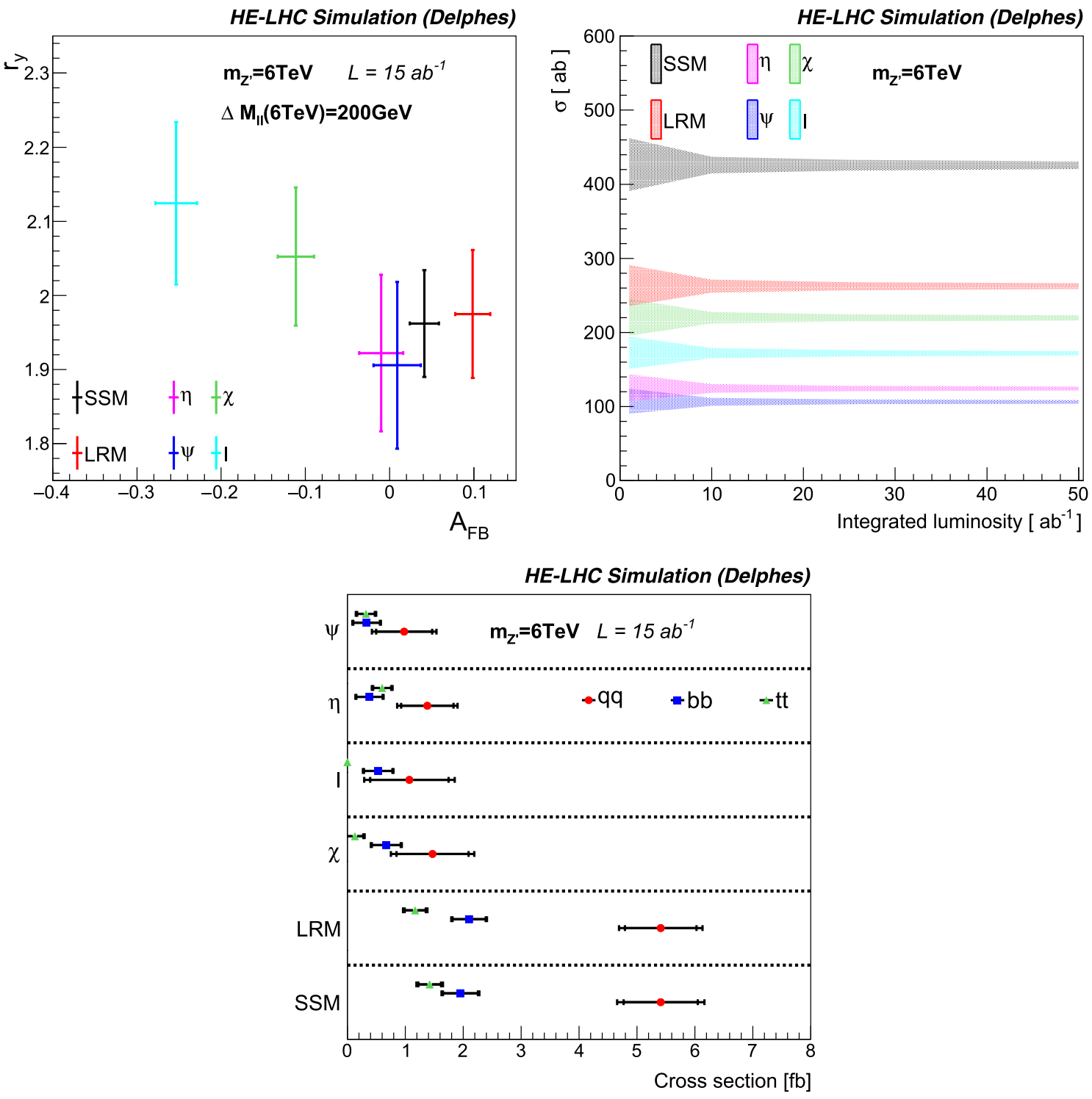

Fig. 12 Left: scatter plot of $r_{y}$ versus $A_{F B}$ with a $200 \mathrm{GeV}$ mass window. The full interference is included. Right: fitted signal cross-section together with its corresponding error versus integrated luminosity. Bot- tom: Fitted cross-section of the three hadronic analyses. Statistical and full uncertainties are shown on each point

dictions for $r_{y}$ and $A_{F B}$, have measurably different fractions of $t \bar{t}$ or $b \bar{b}$ final states. We note however that the degeneracy between $\eta$ and $\psi$ can only be partially resolved at $\approx 1 \sigma$ by exploiting the difference in $t \bar{t}$ yield. We note that ratios of these individual production cross sections are insensitive to the possible existence of other non-SM decay modes of the $Z^{\prime}$.

\section{Conclusions}

This paper had three main goals: (i) to determine the discovery reach of the FCC-hh and HE-LHC at the highest masses, 
using as benchmarks several BSM models of $s$-channel resonance production, (ii) to define performance targets for the detectors, and (iii) to study the power of the HE-LHC to discriminate among different models of resonances that could be just visible at HL-LHC. We confirmed the expectation that the discovery reach scales approximately as the increase in the beam energy: this is not a trivial finding, since the energy measurement and the reconstruction of the multi-TeV decay final states (dileptons or different types of dijets) is not guaranteed, and requires important improvements with respect to the performance of the LHC detectors (e.g. higher calorimeter granularity for the reconstruction and identification of jets, or better momentum resolution for muons). That these improvements are potentially within the reach of foreseeable technology, as indicated by the preliminary detector design proposals for FCC-hh [3], indicates that the FCC-hh physics potential can be fully exploited.

We also studied the discrimination potential of six $Z^{\prime}$ models at HE-LHC. The exercise was performed assuming the evidence of an excess observed at $\sqrt{s}=14 \mathrm{TeV}$ at a mass $m_{Z^{\prime}} \approx 6 \mathrm{TeV}$. Overall it was found that the increased production cross section and the corresponding statistical increase from HL-LHC to HE-LHC are sufficient to analyze an extended set of observables, whose global behaviour provides important information to distinguish among most models. Further studies, using for example 3-body decay modes or associated Z' production (with jets or with SM gauge bosons), could be considered to provide additional handles characterizing the resonance properties.

Acknowledgements We thank Ben Allanach for helpful discussions on the interpretation of the $R_{K^{(*)}}$ anomalies and for providing the "naive" model to be tested in the context of the FCC-hh detector studies. The work of TGR was supported by the Department of Energy, Contract DE-AC02-76SF00515.

Data Availability Statement This manuscript has no associated data or the data will not be deposited. [Authors' comment: The data are not deposited because the studies have been made within the FCC framework, which requires the signature of a Memorandum of Understanding, thus we believe it is better not to deposit them.]

Open Access This article is distributed under the terms of the Creative Commons Attribution 4.0 International License (http://creativecomm ons.org/licenses/by/4.0/), which permits unrestricted use, distribution, and reproduction in any medium, provided you give appropriate credit to the original author(s) and the source, provide a link to the Creative Commons license, and indicate if changes were made. Funded by SCOAP ${ }^{3}$.

\section{Appendix A: Discussion of the detector performance}

In the calorimeters, the energy resolution at high energy is determined by the constant term. The value of the constant term is different for ECAL and HCAL calorimeters. It is ulti- mately determined by the choice of the calorimeter technology and the design. Large constant terms typically originate from inhomogenities among different detector elements and energy leakages due to sub-optimal shower containment. The calorimeters of the FCC-hh detector must therefore be capable of containing EM and hadronic showers in the multi-TeV regime in order to achieve small constant terms. Comparing with the LHC experiments, we require a performance of $\sigma_{E} / E \approx 0.3 \%$ and $\sigma_{E} / E \approx 3 \%$ for the ECAL and HCAL, respectively. As shown in Fig. 13 (left), the effect induced by the magnitude of the hadronic calorimeter constant term on the expected discovery reach for heavy $Z_{\text {SSM }}^{\prime}$ resonances decaying hadronically is sizable. We note that, despite the fraction of electromagnetic energy from $\pi^{0}$ 's large in jets, the sensitivity is entirely driven by the hadronic calorimeter resolution given its worse intrinsic resolution.

Muons cannot be reconstructed with calorimetric methods. ${ }^{4}$ Since the muon momentum is obtained through a fit of the trajectory that uses as input a combination of track and muon spectrometer hits, the muon momentum resolution degrades with increasing momentum, as $\frac{\sigma_{p}}{p}=a \oplus b p$ where $a$ is the constant term determined by the amount of material responsible for multiple scattering in the tracking volume. As with jets, electrons and photons, a good muon momentum resolution at multi- $\mathrm{TeV}$ energy is crucial for maintaining a high sensitivity in searches for heavy new states that might decay to muons. The reach for a $Z^{\prime} \rightarrow \mu \mu$ resonance obtained with various assumptions on the muon resolution is illustrated in Fig. 13 (right). The best sensitivity is achieved with an assumed $\sigma_{p} / p \approx 5 \%$ at $p_{\mathrm{T}}=20 \mathrm{TeV}$ corresponding to our target for the FCC-hh detector, as opposed to the projected CMS resolution of $\sigma_{p} / p \approx 40 \%$. In order to reconstruct and measure accurately the momentum of $p_{\mathrm{T}}=20 \mathrm{TeV}$ a large lever arm is needed and excellent spatial resolution and precise alignment of the tracking plus muon systems is also needed. The specifics of the design that allows to reach such required performance are discussed in Ref. [3].

New heavy states could decay to multi-TeV $c$ and $b$ quarks. FCC-hh detectors must therefore be capable of efficiently identifying multi-TeV long-lived hadrons. A $p_{\mathrm{T}}=$ $5 \mathrm{TeV}$ b-hadron is qualitatively very different from $p_{\mathrm{T}}=$ $100 \mathrm{GeV}$ b-hadron. The latter decays on average within the vertex detector acceptance and can be identified by means of displaced vertex reconstruction. Conversely, the former decays on average at a distance $\gamma c \tau=50 \mathrm{~cm}$, well outside the pixel detector volume. Reconstructing such highly displaced b-jets will require a paradigm shift in heavy flavour

\footnotetext{
4 Calorimetric information can however help for muon identification. For example a $20 \mathrm{TeV}$ muon deposits through radiative energy loss on average $\Delta E=200 \mathrm{GeV}$ in $3 \mathrm{~m}$ of iron, corresponding to $1 \%$ of the initial muon energy.
} 

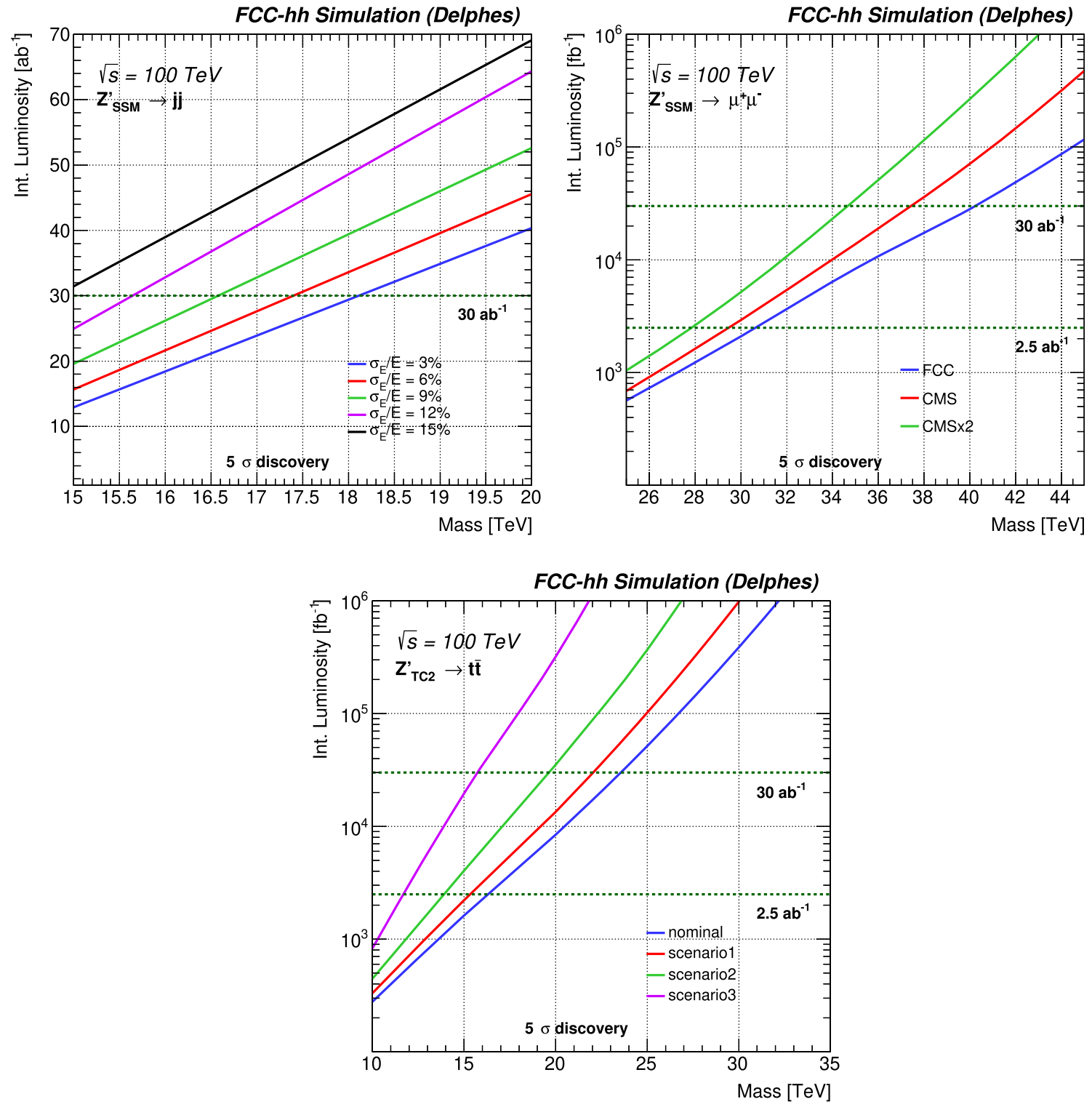

Fig. 13 Luminosity versus mass for a $5 \sigma$ discovery for different calorimeter resolution (left), muon resolutions (right) and b-tagging scenarios (bottom)

reconstruction. The success of algorithms exploring large hit multiplicity discontinuities among subsequent tracking layer heavily relies on excellent granularity of the tracking system, in both longitudinal and transverse directions. High efficiencies $\left(\epsilon_{b}>60 \%\right)$ for corresponding low mis-identification probability $\left(\epsilon_{u, d, s}<1 \%\right)$ from light jets have to be achieved up to $p_{\mathrm{T}}=5 \mathrm{TeV}$. For example, searches for heavy resonances decaying to hadronic $t \bar{t}$ pairs heavily rely on efficient b-tagging performance at such energies. The discovery reach for a specific $Z^{\prime}$ model assuming several scenarios for b-jet identification at high energies is shown in Fig. 13 bottom. Various scenarios of b-tagging efficiencies at very large $p_{\mathrm{T}}$ are considered. The nominal efficiency is given in Table 3, and scenarios 1, 2 and 3 correspond to reduction of the slope respectively by a factor $25 \%, 33 \%$ and $50 \%$. As expected the discovery reach strongly depends on the b-tagging performances.

\section{Appendix B: Tagging rate function}

Given a jet with specific values of $\eta, p_{\mathrm{T}}$ and with flavour $f$, its tagging probability can be denoted as:

$\varepsilon\left(f,|\eta|, p_{\mathrm{T}}\right)$.

For a given event with $N$ jets, its probability of containing exactly one $b$-tag jet can be computed as: 

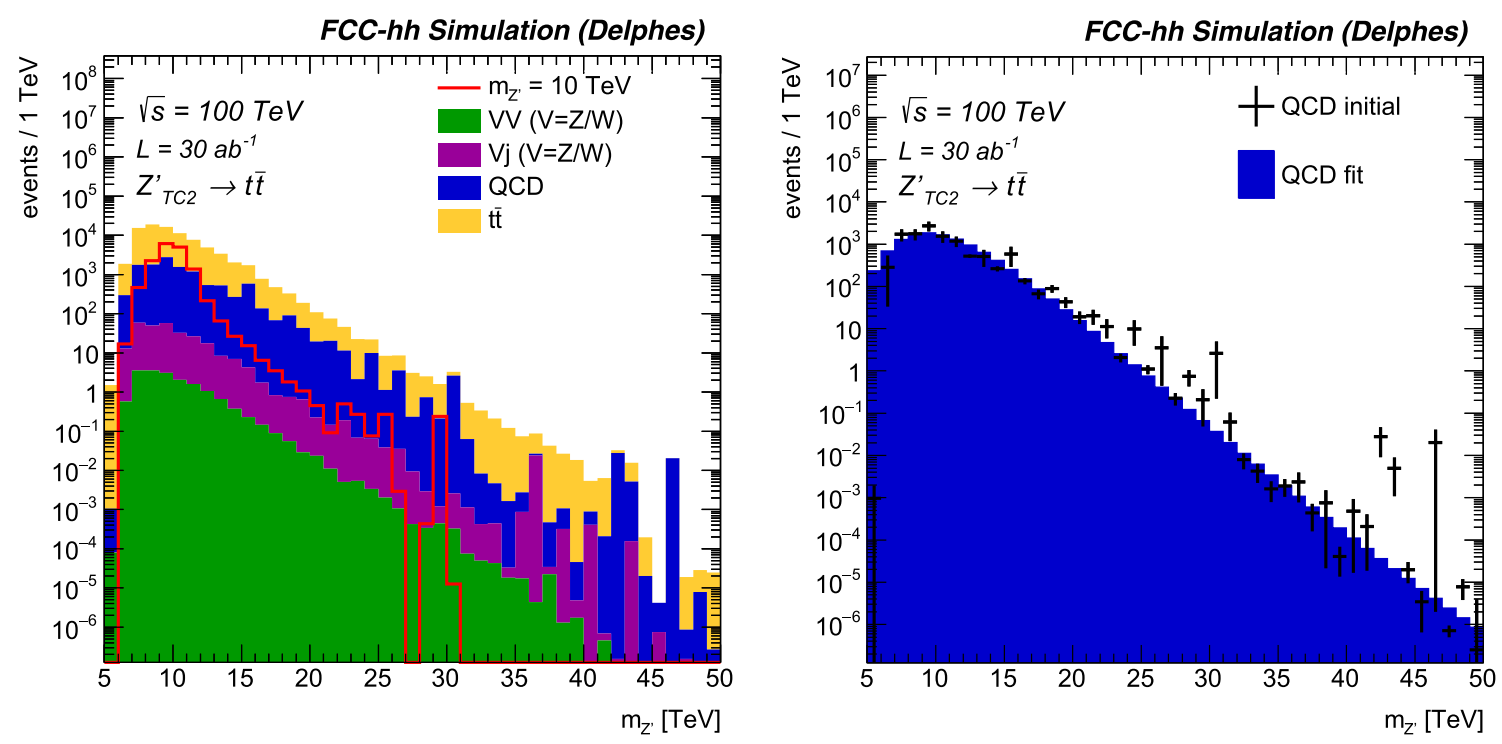

Fig. 14 QCD Background invariant mass spectra prior to fit

$P_{=1}=\sum_{i=1}^{N}\left(\varepsilon_{i} \prod_{j \neq i}\left(1-\varepsilon_{j}\right)\right)$.

In the same way, it can be used to compute the probability for inclusive $b$-tag selections:

$P=0=\prod_{j=1}^{N}\left(1-\varepsilon_{j}\right)$

$P_{\geq 1}=1-P_{=0}$.

It was verified that the TRF methods agree well with the direct tagging.

\section{Appendix C: Background fit}

The function used to fit the background shapes when Monte Carlo statistics are insufficient can be expressed as:

$f(z)=p_{1}(1-z)^{p_{2}} z^{p_{3}} z^{p_{4} \log z}$,

where $z=m_{j j} / \sqrt{s}$, with $m_{j j}$ the invariant mass of the two highest energetic objects and $\sqrt{s}$ the center of mass energy. Fitting the invariant mass distribution with the function 7.1 allows to obtain a smooth shape, while the overall normalisation is taken prior to the fit. Figure 14 (left) shows the $Z^{\prime} \rightarrow t \bar{t}$ invariant mass distribution after the final selection for the various backgrounds and a $10 \mathrm{TeV}$ signal. Large statistical fluctuations can be observed especially for the QCD background that is heavily suppressed thanks to the multivariate object tagger. The right panel represents the same QCD invariant mass distribution before the fit (dots) and after the

fit (plain). Good agreement is observed and the fitted distribution normalised to the pre-fit yields is used to obtain the results in the statistical analysis.

\section{Appendix D: Multivariate object tagger}

The training samples are built from $Z^{\prime}$ samples with $m_{Z^{\prime}}=$ $20 \mathrm{TeV}$ using jets that do not contain leptons. The $E_{F}(n=$ $1, \alpha=0.05)$ observable used as input to the $W$-tagger is shown in Fig. 15 (left) and the $\tau_{32}$ observable used as input to the top-tagger in Fig. 15 (right). The evolution of the light (u,d, and s quark) flavour jet efficiency (mis-tag rate) versus the $W$ and top tagging efficiencies for both taggers is shown in Fig. 15 (bottom). Several cross-checks have been performed to further validate the multivariate training procedure. By removing highly correlated variables it has been checked that the same performance is achieved. In addition, the BDT response has been tested with different signal masses. For the selection used in the analysis (BDT score greater than 0.15), the shape of the BDT does not significantly impact the signal efficiency. The list of input variables used to train the BDT, ordered by the training weight, can be found in Table 8.

\section{Appendix E: Summary plots}

The discovery potential (top) and 95\% CL limits (bottom) for the heavy resonances presented in this document are summarised in Fig. 16 for FCC-hh (left) and HE-LHC (right). 

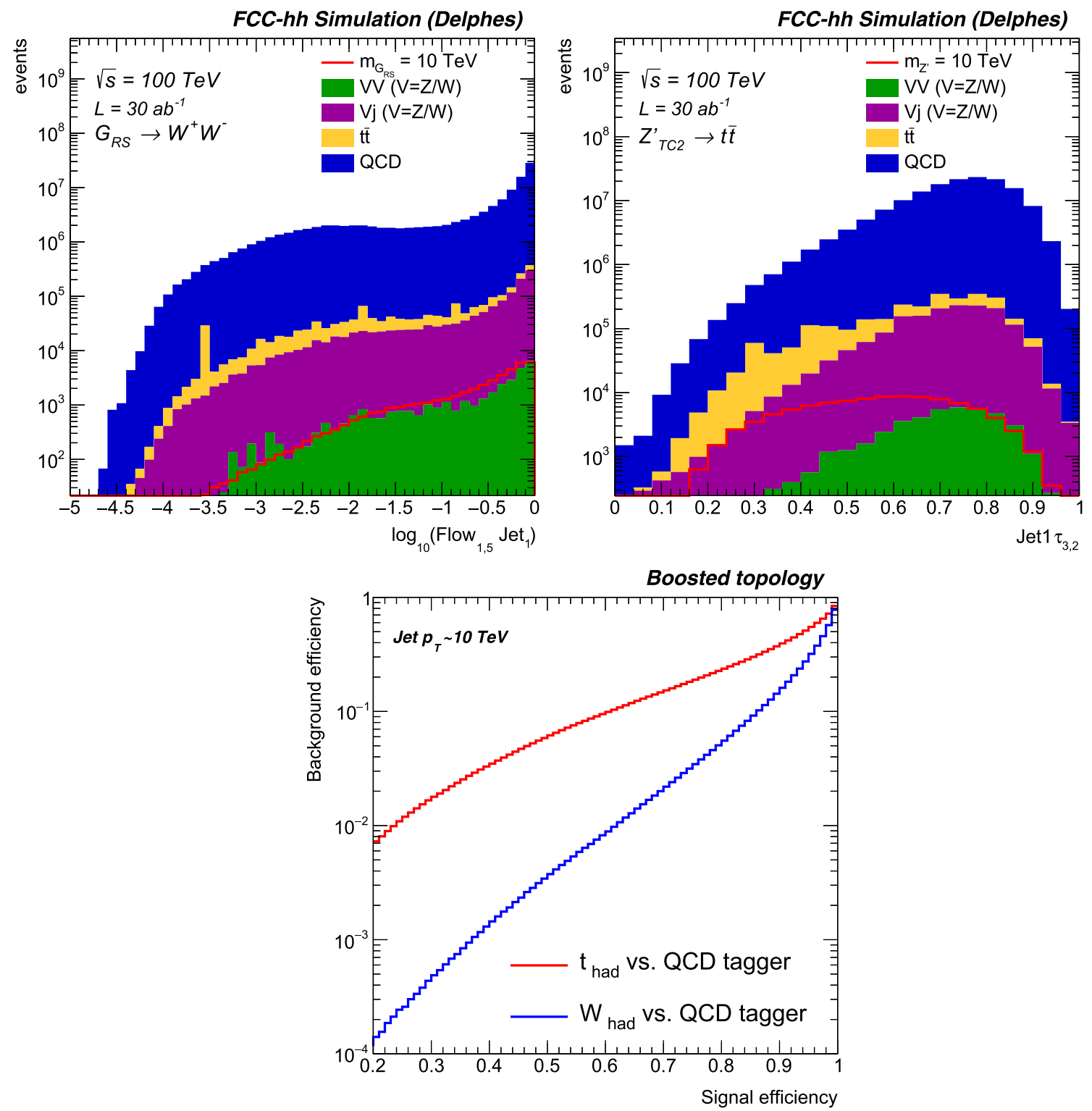

Fig. 15 Left: energy-flow $E_{F}(n=1, \alpha=0.05)$ observable for the leading jet $p_{\mathrm{T}}$ of the $G_{R S} \rightarrow W^{+} W^{-}$analysis at the pre-selection level (two high $p_{\mathrm{T}}$ jets). Right: $\tau_{32}$ observable for the leading jet $p_{\mathrm{T}}$ of the $Z^{\prime} \rightarrow t \bar{t}$ analysis at the pre-selection level (two high $p_{\mathrm{T}}$ jets). Bottom: light jet rejection versus tagging efficiency for the $W$-tagger (blue) and top-tagger (red)
Table 8 Summary of the input variables to the BDT and their relative weight for both $W$ and top taggers

\begin{tabular}{|c|c|c|c|}
\hline \multicolumn{2}{|l|}{$W$ tagger } & \multicolumn{2}{|l|}{ Top tagger } \\
\hline Variable & Weight & Variable & Weight \\
\hline$\tau_{3}($ track jet, $\mathrm{R}=0.2)$ & 0.12 & $\tau_{1}($ track jet, $\mathrm{R}=0.2)$ & 0.21 \\
\hline$m_{S D}($ track jet, $\mathrm{R}=0.2)$ & 0.11 & $m_{S D}($ track jet, $\mathrm{R}=0.2)$ & 0.17 \\
\hline$\tau_{31}($ track jet, $R=0.2)$ & 0.10 & $\tau_{31}($ track jet, $\mathrm{R}=0.2)$ & 0.11 \\
\hline$E_{F}(n=5, \alpha=0.05)$ & 0.09 & $\tau_{2}($ track jet, $R=0.2)$ & 0.10 \\
\hline$E_{F}(n=4, \alpha=0.05)$ & 0.09 & $\tau_{3}($ track jet, $\mathrm{R}=0.2)$ & 0.09 \\
\hline$E_{F}(n=1, \alpha=0.05)$ & 0.08 & $m_{S D}($ track jet, $\mathrm{R}=0.8)$ & 0.09 \\
\hline$E_{F}(n=2, \alpha=0.05)$ & 0.07 & $m_{S D}($ track jet, $\mathrm{R}=0.4)$ & 0.09 \\
\hline
\end{tabular}


Table 8 continued

\begin{tabular}{|c|c|c|c|}
\hline \multicolumn{2}{|l|}{$W$ tagger } & \multicolumn{2}{|l|}{ Top tagger } \\
\hline Variable & Weight & Variable & Weight \\
\hline$E_{F}(n=3, \alpha=0.05)$ & 0.06 & $\tau_{32}($ track jet, $\mathrm{R}=0.2)$ & 0.08 \\
\hline$\tau_{21}($ track jet, $R=0.2)$ & 0.06 & $\tau_{21}($ track jet, $R=0.2)$ & 0.06 \\
\hline$m_{S D}($ track jet, $\mathrm{R}=0.8)$ & 0.06 & & \\
\hline$m_{S D}($ track jet, $\mathrm{R}=0.4)$ & 0.06 & & \\
\hline$\tau_{1}($ track jet, $\mathrm{R}=0.2)$ & 0.05 & & \\
\hline$\tau_{2}($ track jet, $\mathrm{R}=0.2)$ & 0.04 & & \\
\hline$\tau_{32}($ track jet, $R=0.2)$ & 0.02 & & \\
\hline
\end{tabular}
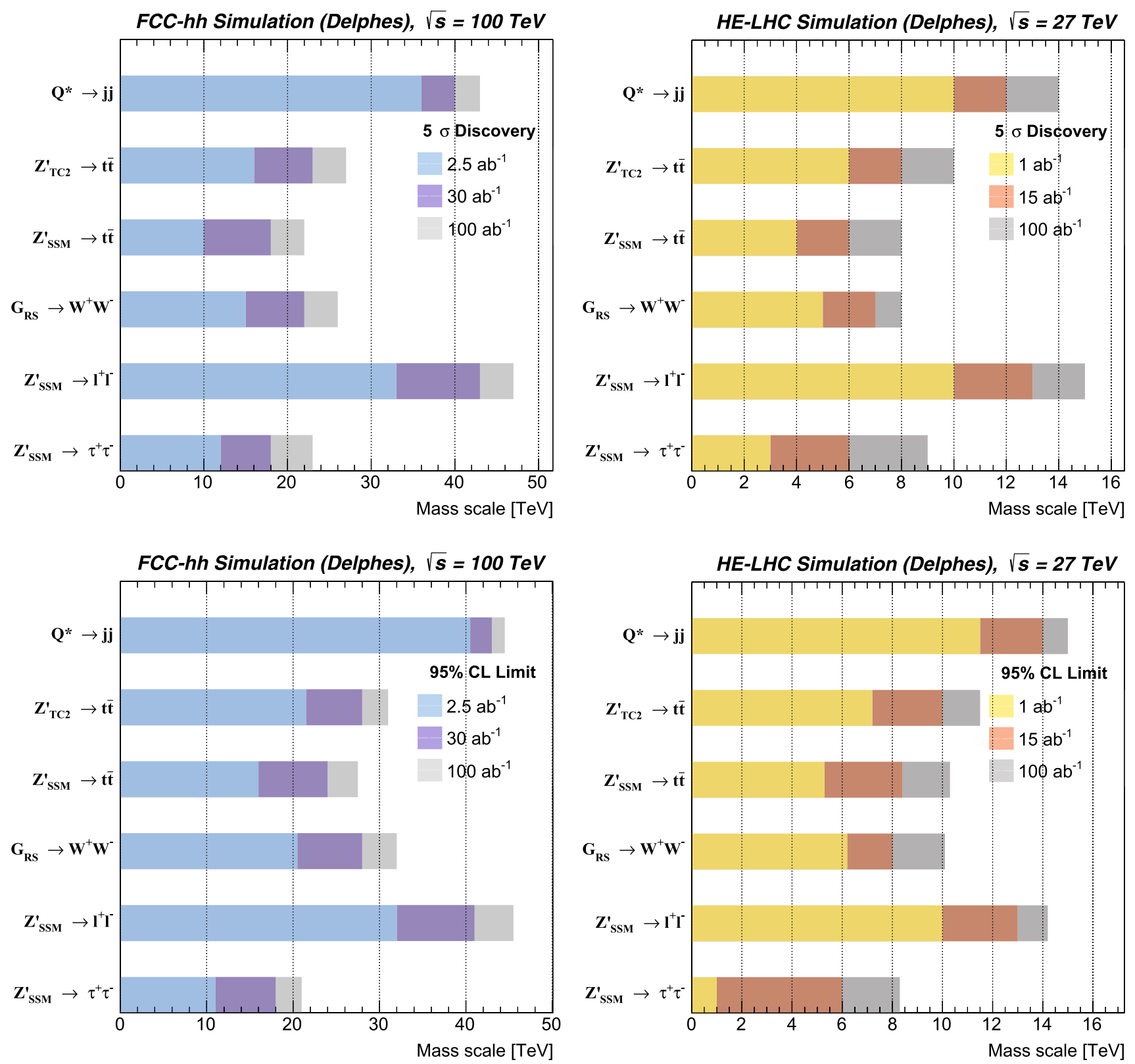

Fig. 16 Summary of a $5 \sigma$ discovery reach (top) and 95\% CL limits (bottom) as a function of the resonance mass for different luminosity scenarios of FCC-hh (left) and HE-LHC (right) 


\section{References}

1. X. Cid Vidal et al, Beyond the Standard Model Physics at the HLLHC and HE-LHC, arXiv:1812.07831 [hep-ph]

2. F. Zimmermann, M. Benedikt, M. Capeans Garrido, F. Cerutti, B. Goddard, J. Gutleber et al., Future Circular Collider Study. Volume 4: The High Energy LHC (HE-LHC). Tech. Rep. CERN-ACC2018-0059, CERN, Geneva (2018). https://cds.cern.ch/record/ 2651305

3. M. Benedikt, M. Capeans Garrido, F. Cerutti, B. Goddard, J. Gutleber, J.M. Jimenez et al., Future Circular Collider Study. Volume 3: The Hadron Collider (FCC-hh). Tech. Rep. CERN-ACC-20180058, CERN, Geneva (2018). https://cds.cern.ch/record/2651300

4. M. Mangano, P. Azzi, M. Benedikt, A. Blondel, D.A. Britzger, A. Dainese et al., Future Circular Collider Study. Volume 1: Physics Opportunities. Tech. Rep. CERN-ACC-2018-0056, CERN, Geneva (2018). https://cds.cern.ch/record/2651294

5. CEPC STUDY GROUP collaboration, CEPC conceptual design report: volume 1-accelerator. arXiv:1809.00285 [physics.acc-ph]

6. CEPC STUDY GROUP collaboration, CEPC conceptual design report: volume 2-physics \& detector. arXiv: 1811.10545 [hep-ex]

7. T. Golling et al., Physics at a $100 \mathrm{TeV}$ pp collider: beyond the Standard Model phenomena. CERN Yellow Report (2017) 441. arXiv:1606.00947 [hep-ph]

8. P. Langacker, The physics of heavy $Z^{\prime}$ gauge bosons. Rev. Mod. Phys. 81, 1199 (2009). arXiv:0801.1345 [hep-ph]

9. T. G. Rizzo, $Z^{\prime}$ phenomenology and the LHC, in Proceedings of Theoretical Advanced Study Institute in Elementary Particle Physics : Exploring New Frontiers Using Colliders and Neutrinos (TASI 2006): Boulder, Colorado, June 4-30, 2006, pp. 537-575, (2006) arXiv:hep-ph/0610104, http://www-public.slac.stanford. edu/sciDoc/docMeta.aspx?slacPubNumber=slac-pub-12129

10. M. Carena, A. Daleo, B.A. Dobrescu, T.M.P. Tait, $Z^{\prime}$ gauge bosons at the Tevatron. Phys. Rev. D 70, 093009 (2004). arXiv:hep-ph/0408098

11. E. Salvioni, G. Villadoro, F. Zwirner, Minimal Z-prime models: present bounds and early LHC reach. JHEP 11, 068 (2009). arXiv:0909.1320 [hep-ph]

12. G. Senjanovic, R.N. Mohapatra, Exact left-right symmetry and spontaneous violation of parity. Phys. Rev. D 12, 1502 (1975)

13. R.N. Mohapatra, G. Senjanovic, Neutrino masses and mixings in gauge models with spontaneous parity violation. Phys. Rev. D 23, $165(1981)$

14. R.W. Robinett, J.L. Rosner, Mass scales in grand unified theories. Phys. Rev. D 26, 2396 (1982)

15. D. London, J.L. Rosner, Extra gauge bosons in E(6). Phys. Rev. D 34, 1530 (1986)

16. J.L. Hewett, T.G. Rizzo, Low-energy phenomenology of superstring inspired E(6) models. Phys. Rep. 183, 193 (1989)

17. A. Joglekar, J.L. Rosner, Searching for signatures of $E_{6}$. Phys. Rev. D 96, 015026 (2017). arXiv:1607.06900 [hep-ph]

18. F. del Aguila, M. Cvetic, P. Langacker, Determination of Z-prime gauge couplings to quarks and leptons at future hadron colliders. Phys. Rev. D 48, R969 (1993). arXiv:hep-ph/9303299

19. G. Altarelli, B. Mele, M. Ruiz-Altaba, Searching for new heavy vector bosons in $p \bar{p}$ colliders. Z. Phys. C 45, 109 (1989)

20. C.T. Hill, Topcolor assisted technicolor. Phys. Lett. B 345, 483 (1995). arXiv:hep-ph/9411426

21. N. Arkani-Hamed, A.G. Cohen, H. Georgi, Electroweak symmetry breaking from dimensional deconstruction. Phys. Lett. B 513, 232 (2001). arXiv:hep-ph/0105239

22. LHCB collaboration, R. Aaij et al., Test of lepton universality using $B^{+} \rightarrow K^{+} \ell^{+} \ell^{-}$decays. Phys. Rev. Lett. 113, 151601 (2014). arXiv:1406.6482 [hep-ex]
23. LHCB collaboration, R. Aaij et al., Test of lepton universality with $B^{0} \rightarrow K^{* 0} \ell^{+} \ell^{-}$decays. JHEP 08, 055 (2017). arXiv: 1705.05802 [hep-ex]

24. S. Bifani, S. Descotes-Genon, A. Romero Vidal, M.-H. Schune, Review of Lepton Universality tests in $B$ decays. arXiv:1809.06229 [hep-ex]

25. B.C. Allanach, B. Gripaios, T. You, The case for future hadron colliders from $B \rightarrow K^{(*)} \mu^{+} \mu^{-}$decays. JHEP 03, 021 (2018). arXiv:1710.06363 [hep-ph]

26. B.C. Allanach, T. Corbett, M.J. Dolan, T. You, Hadron collider sensitivity to fat flavourful $Z^{\prime}$ s for $R_{K^{(*)}}$. arXiv:1810.02166 [hep$\mathrm{ph}]$

27. U. Baur, I. Hinchliffe, D. Zeppenfeld, Excited quark production at hadron colliders. Int. J. Mod. Phys. A 2, 1285 (1987)

28. U. Baur, M. Spira, P.M. Zerwas, Excited quark and lepton production at hadron colliders. Phys. Rev. D 42, 815 (1990)

29. R.M. Harris, K. Kousouris, Searches for dijet resonances at hadron colliders. Int. J. Mod. Phys. A 26, 5005 (2011). arXiv:1110.5302 [hep-ex]

30. N. Boelaert, T. Akesson, Dijet angular distributions at $\mathrm{s}^{* *}(1 / 2)=14$ TeV. Eur. Phys. J. C 66, 343 (2010). arXiv:0905.3961 [hep-ph]

31. R. Sekhar Chivukula, E.H. Simmons, N. Vignaroli, Distinguishing dijet resonances at the LHC. Phys. Rev. D 91, 055019 (2015). arXiv: 1412.3094 [hep-ph]

32. R.S. Chivukula, K.A. Mohan, D. Sengupta, E.H. Simmons, Characterizing boosted dijet resonances with energy correlation functions. JHEP 03, 133 (2018). arXiv:1710.04661 [hep-ph]

33. L. Randall, R. Sundrum, A large mass hierarchy from a small extra dimension. Phys. Rev. Lett. 83, 3370 (1999). arXiv:hep-ph/9905221

34. A. Pomarol, Gauge bosons in a five-dimensional theory with localized gravity. Phys. Lett. B 486, 153 (2000). arXiv:hep-ph/9911294

35. H. Davoudiasl, J.L. Hewett, T.G. Rizzo, Bulk gauge fields in the Randall-Sundrum model. Phys. Lett. B 473, 43 (2000). arXiv:hep-ph/9911262

36. Y. Grossman, M. Neubert, Neutrino masses and mixings in nonfactorizable geometry. Phys. Lett. B 474, 361 (2000). arXiv:hep-ph/9912408

37. H. Davoudiasl, J.L. Hewett, T.G. Rizzo, Experimental probes of localized gravity: on and off the wall. Phys. Rev. D 63, 075004 (2001). arXiv:hep-ph/0006041

38. T. Gherghetta, A. Pomarol, Bulk fields and supersymmetry in a slice of AdS. Nucl. Phys. B 586, 141 (2000). arXiv:hep-ph/0003129

39. H. Davoudiasl, J.L. Hewett, T.G. Rizzo, Phenomenology of the Randall-Sundrum gauge hierarchy model. Phys. Rev. Lett. 84, 2080 (2000). arXiv:hep-ph/9909255

40. T. Sjöstrand, S. Ask, J .R. Christiansen, R. Corke, N. Desai, P. Ilten et al., An introduction to PYTHIA 8.2. Comput. Phys. Commun 191, 159 (2015). arXiv:1410.3012 [hep-ph]

41. NNPDF collaboration, R. D. Ball et al., Parton distributions for the LHC Run II, JHEP 04040 (2015) arXiv:1410.8849 [hep-ph]

42. J. Alwall, R. Frederix, S. Frixione, V. Hirschi, F. Maltoni, O. Mattelaer et al., The automated computation of tree-level and nextto-leading order differential cross sections, and their matching to parton shower simulations. JHEP 07, 079 (2014). arXiv: 1405.0301 [hep-ph]

43. DELPHES 3 collaboration, J. de Favereau, C. Delaere, P. Demin, A. Giammanco, V. Lemaître, A. Mertens et al., DELPHES 3, A modular framework for fast simulation of a generic collider experiment, JHEP 02057 (2014) arXiv:1307.6346 [hep-ex]

44. FCC-hh detector DELPHES card. https://github.com/delphes/ delphes/blob/master/cards/FCC/FCChh.tcl

45. HE/HL-LHC detector DELPHES card. https://github.com/ delphes/delphes/blob/master/cards/delphes_card_HLLHC.tcl

46. ATLAS collaboration, G. Aad et al., The ATLAS Experiment at the CERN Large Hadron Collider, JINST 3 S08003 (2008) 
47. ATLAS collaboration, M. Capeans, G. Darbo, K. Einsweiler, M. Elsing, T. Flick, M. Garcia-Sciveres et al., ATLAS Insertable B-Layer Technical Design Report

48. CMS collaboration, S. Chatrchyan et al., The CMS experiment at the CERN LHC. JINST 3, S08004 (2008)

49. CMS collaboration, D.A. Matzner Dominguez, D. Abbaneo, K. Arndt, N. Bacchetta, A. Ball, E. Bartz et al., CMS Technical Design Report for the Pixel Detector Upgrade

50. GEANT4 collaboration, S. Agostinelli et al., GEANT4: a simulation toolkit. Nucl. Instrum. Methods A 506, 250 (2003)

51. J. Allison et al., Geant 4 developments and applications. IEEE Trans. Nucl. Sci. 53, 270 (2006)

52. J. Allison et al., Recent developments in Geant4. Nucl. Instrum. Methods A 835, 186 (2016)

53. M. Cacciari, G.P. Salam, G. Soyez, FastJet user manual. Eur. Phys. J. C 72, 1896 (2012). arXiv:1111.6097 [hep-ph]

54. M. Cacciari, G.P. Salam, G. Soyez, The anti- $k_{t}$ jet clustering algorithm. JHEP 04, 063 (2008). arXiv:0802.1189 [hep-ex]

55. J. Thaler, K. Van Tilburg, Identifying boosted objects with $\mathrm{N}$ subjettiness. JHEP 03, 015 (2011). arXiv: 1011.2268 [hep-ph]

56. A.J. Larkoski, S. Marzani, G. Soyez, J. Thaler, Soft drop. JHEP 05, 146 (2014). arXiv:1402.2657 [hep-ph]

57. C. H. Yeh, S. V. Chekanov, A. V. Kotwal, J. Proudfoot, S. Sen, N. V. Tran et al., Studies of granularity of a hadronic calorimeter for tens-of-TeV jets at a $100 \mathrm{TeV} p p$ collider, arXiv:1901.11146 [hep-ex]

58. E. Perez Codina, P.G. Roloff, Hit multiplicity approach to b-tagging in FCC-hh. Tech. Rep. CERN-ACC-2018-0023, CERN, Geneva (2018). https://cds.cern.ch/record/2631478

59. E. Perez Codina, P.G. Roloff, Tracking and Flavour Tagging at FCC-hh. Tech. Rep. CERN-ACC-2018-0027, CERN, Geneva (2018). https://cds.cern.ch/record/2635893

60. T. Junk, Confidence level computation for combining searches with small statistics. Nucl. Instrum. Methods A 434, 435 (1999). arXiv:hep-ex/9902006

61. A.L. Read, Presentation of search results: the CL(s) technique. J. Phys. G 28, 2693 (2002)

62. G. Cowan, K. Cranmer, E. Gross, O. Vitells, Asymptotic formulae for likelihood-based tests of new physics. Eur. Phys. J. C 71, 1554 (2011). arXiv:1007.1727 [physics.data-an]
63. CMS collaboration, V. Khachatryan et al., Search for heavy resonances decaying to tau lepton pairs in proton-proton collisions at $\sqrt{s}=13$ TeV. JHEP 02, 048 (2017). arXiv:1611.06594 [hep-ex]

64. G. D’Amico, M. Nardecchia, P. Panci, F. Sannino, A. Strumia, R. Torre et al., Flavour anomalies after the $R_{K^{*}}$ measurement. JHEP 09, 010 (2017). arXiv:1704.05438 [hep-ph]

65. A.J. Larkoski, F. Maltoni, M. Selvaggi, Tracking down hyperboosted top quarks. JHEP 06, 032 (2015). arXiv: 1503.03347 [hep$\mathrm{ph}]$

66. M.L. Mangano et al., Physics at a $100 \mathrm{TeV}$ pp collider: standard model processes. CERN Yellow Report 1 (2017). arXiv:1607.01831 [hep-ph]

67. B. Auerbach, S. Chekanov, J. Love, J. Proudfoot, A.V. Kotwal, Sensitivity to new high-mass states decaying to $t \bar{t}$ at a $100 \mathrm{TeV}$ collider. Phys. Rev. D 91, 034014 (2015). arXiv:1412.5951 [hep$\mathrm{ph}]$

68. D. Liu, L.-T. Wang, K.-P. Xie, Prospects of searching for composite resonances at the LHC and beyond. JHEP 01, 157 (2019). arXiv:1810.08954 [hep-ph]

69. S.V. Chekanov, J.T. Childers, J. Proudfoot, D. Frizzell, R. Wang, Precision searches in dijets at the HL-LHC and HE-LHC. JINST 13, P05022 (2018). arXiv:1710.09484 [hep-ex]

70. T.G. Rizzo, Exploring new gauge bosons at a $100 \mathrm{TeV}$ collider. Phys. Rev. D 89, 095022 (2014). arXiv:1403.5465 [hep-ph]

71. T. Han, P. Langacker, Z. Liu, L.-T. Wang, Diagnosis of a new neutral gauge boson at the LHC and ILC for snowmass (2013). arXiv:1308.2738 [hep-ph]

72. L.A. Harland-Lang, A.D. Martin, P. Motylinski, R.S. Thorne, Parton distributions in the LHC era: MMHT 2014 PDFs. Eur. Phys. J. C 75, 204 (2015). arXiv:1412.3989 [hep-ph]

73. ATLAS collaboration, M. Aaboud et al., Search for new high-mass phenomena in the dilepton final state using $36 \mathrm{fb}^{-1}$ of protonproton collision data at $\sqrt{s}=13 \mathrm{TeV}$ with the ATLAS detector. JHEP 10182 (2017). arXiv:1707.02424 [hep-ex]

74. CMS collaboration, A.M. Sirunyan et al., Search for high-mass resonances in dilepton final states in proton-proton collisions at $\sqrt{s}=13$ TeV. JHEP 06, 120 (2018). arXiv:1803.06292 [hep-ex] 